\title{
EFFECTIVE ELASTICITY COEFFICIENTS IN DRY POROUS MATERIALS. NUMERICAL AND SEMI-ANALYTICAL APPROACHES
}

\author{
Vladimir Sladek $^{1}$, Bruno Musil ${ }^{2}$, and Jan Sladek ${ }^{1}$ \\ ${ }^{1}$ Institute of Construction and Architecture, Slovak Academy of Sciences \\ 84503 Bratislava, Slovakia \\ e-mail: vladimir.sladek@savba.sk,jan.sladek@savba.sk \\ ${ }^{2}$ Institute of Mechanics, Bundeswehr University Munich \\ 85579 Neubiberg, Germany \\ bruno.musil@gmail.com
}

Keywords: Empty pores, Homogenization, Representative volume elements, Micro-structural boundary value problems, Numerical experiments.

\begin{abstract}
The paper deals with homogenization of linear elastic continuum involving empty pores. We concern some aspects of numerical approaches and confront them with the analytical ones in case of $2 \mathrm{D}$ elasticity. Of course, there are some restrictions for the analytical concept, because it is applicable only to few shapes of pores. Nevertheless, two or four variants of the analytical approach are developed in order to study the role of incorporation of interaction among the pores as well as the influence of outer boundary of microstructural subdomain and application of various physically admissible correlations between the volume averages for homogenized continuum and micro-structural one. In general, a numerical approach is necessary for solution of micro-structural boundary value problems in case of arbitrary shape and/or distribution of voids. Two approaches are proposed and mathematical models developed for numerical calculation of effective material coefficients for linear elastic continuum involving arbitrary empty pores. Appropriate micro-structural boundary value problems in the RVE are proposed for numerical analyses which are utilized for a posterior evaluation of effective material coefficients. Comparisons of results by the analytical and numerical approaches are discussed for the circular and elliptical pores. Finally, the influence of the shape of vacant pores and porosity on bending of elastic plates is illustrated in numerical simulations.
\end{abstract}




\section{INTRODUCTION}

In practical engineering problems, the material media are not often homogeneous. If the size of material defects is much smaller than the characteristic length in structural design, the concept of homogenization is meaningful and applicable. Since the design is mostly the matter of numerical simulations, it is important to deal well with evaluation of effective material coefficients. Recently also multiscale models are utilized with different physical treatment on different length scales. In this paper, we shall distinguish between the macro-structure and micro-structure with using the linear elasticity as the physical base for description of phenomena on both dimensional scales. The difference consists in modelling micro-heterogeneities in micro-structure and characterization of macro-structure as statistically homogeneous linear elastic composite or defected materials based on the macroscopic or overall or equivalent elastic behavior. Although the subject of homogenization is classical (Voigt and Reuss mixture rules, Hashin and Shtrikman upper and lower bounds [2], self-consistency method [3], Mori-Tanaka method [4-6, 1]), there are still some open questions [7], e.g. the unproved Hill's and Mandel's conjecture statement.

\section{HOMOGENIZATION BASED ON MICRO-STRUCTURAL MODELLING. EFFECTIVE MATERIAL COEFFICIENTS}

The idea of homogenization is applicable to microscopically inhomogeneous materials only if these materials are macroscopically or statistically homogeneous in considered macrostructure. It means that there exist representative elements (RVEs) of the body under consideration. The RVE must obey the following requirements: (i) it is relatively small sample of the material, i.e. the constraints and loading on the surface of the macrostructure are uniform within the length $l$ which is the linear size of the RVE $(l \ll L$ where $L$ is the characteristic dimension of the macrostructure); (ii) it is sufficiently large as compared with the linear size of micro-inhomogeneity $(a)$ in order the spatial wave-length $(\sim a)$ of the stress and strain fluctuations about a mean value be small compared with $l(a \ll l)$, and the effects of such fluctuations become insignificant within a few wave-lengths from the boundary of the RVE. Having solved micro-structural boundary value problems in RVE (the microconstituents are assumed to be homogeneous elastic continua; the shape and distribution of micro-constituents or defects are abstracted from experiment), one can get volume averages of micro-fields over the RVE. The transition from micro- to macro-level (where the macrostructural problems could be solved in effective continuum which is macroscopically homogeneous) depends on finding the connections between suitably defined macro-variables and averages of micro-fields. There are two main questions: (i) how to define macro-variables and boundary data for the RVE in a physically meaningful way; (ii) whether and how the macrovariables (alone or in combinations) are related to the volume averages of their microcounterparts.

Let us consider a macroscopically homogeneous body and denote by $B$ the regular subregion occupied by a RVE of the same microscopically inhomogeneous $2 \mathrm{D}$ body composed of the homogeneous skeleton $\Omega \subset B$ and empty voids $B-\Omega$ with $\partial B=\Gamma, \partial \Omega=\Gamma \cup \Gamma_{0}, \Gamma_{0}$ being the boundary of the RVE, skeleton and voids in the RVE, respectively. The volume average or the mean values of the field variables are

$$
\left\langle f^{e f f}\right\rangle_{B}=\frac{1}{|B|} \int_{B} f^{e f f}(\mathbf{x}) d \Omega, \quad\left\langle f^{m s}\right\rangle_{\Omega}=\frac{1}{|\Omega|} \int_{\Omega} f^{m s}(\mathbf{x}) d \Omega .
$$

where the former relationship is for the effective field variable $f^{\text {eff }}(\mathbf{x})$ defined in the whole $B$, while the latter is used for the field variable $f^{m s}(\mathbf{x})$ related to the elastic skeleton (matrix mate- 
rial) in micro-structural boundary value problems $(m s-b v p)$. The mean values without detailed specification will be denoted as $\langle f\rangle_{V}$.

If $\left[u_{i}, \varepsilon_{i j}, \sigma_{i j}\right]$ are displacements, corresponding strains and self-equilibrated stress fields corresponding to solution of an elastic boundary value problem in $V$ with boundary $\partial V$ and tractions $t_{i}=\sigma_{i j} n_{j}$ ( $\mathbf{n}$ is the outward unit normal vector on $\partial V$ ), then it can be shown

$$
\begin{aligned}
& |V|\left\langle\sigma_{i j} \varepsilon_{i j}\right\rangle_{V}=\int_{\partial V} t_{i} u_{i} d \Gamma \\
& \int_{\partial V} t_{i} x_{j} d \Gamma=|V|\left\langle\sigma_{i j}\right\rangle_{V}=|V|\left\langle\sigma_{j i}\right\rangle_{V}=\int_{\partial V} t_{j} x_{i} d \Gamma, \quad \frac{1}{2} \int_{\partial V}\left(t_{i} x_{j}+t_{j} x_{i}\right) d \Gamma=|V|\left\langle\sigma_{i j}\right\rangle_{V} \\
& \int_{\partial V} u_{i} n_{j} d \Gamma=|V|\left\langle u_{i, j}\right\rangle_{V}, \quad \frac{1}{2} \int_{\partial V}\left(u_{i} n_{j}+u_{j} n_{i}\right) d \Gamma=|V|\left\langle\varepsilon_{i j}\right\rangle_{V} \\
& |V|\left(\left\langle\sigma_{i j} \varepsilon_{i j}\right\rangle_{V}-\left\langle\sigma_{i j}\right\rangle_{V}\left\langle\varepsilon_{i j}\right\rangle_{V}\right)=\int_{\partial V}\left(t_{i}-n_{k}\left\langle\sigma_{i k}\right\rangle_{V}\right)\left(u_{i}-x_{j}\left\langle\varepsilon_{i j}\right\rangle_{V}\right) d \Gamma
\end{aligned}
$$

Thus, the mean value of the deformation energy can be written in the separated view

$$
\left\langle\sigma_{i j} \varepsilon_{i j}\right\rangle_{V}=\left\langle\sigma_{i j}\right\rangle_{V}\left\langle\varepsilon_{i j}\right\rangle_{V}
$$

if the Hill conditions [ ] are satisfied:

(i) kinematically uniform boundary conditions (KUBC): $\left.u_{i}\right|_{\partial V}=x_{j} \bar{\varepsilon}_{i j}, \quad \bar{\varepsilon}_{i j}=$ const , when $\left\langle\varepsilon_{i j}\right\rangle_{V}=\bar{\varepsilon}_{i j}$

(ii) statically uniform boundary conditions (SUBC): $\left.t_{i}\right|_{\partial V}=n_{k} \bar{\sigma}_{i k}, \bar{\sigma}_{i k}=$ const, when $\left\langle\sigma_{i k}\right\rangle_{V}=\bar{\sigma}_{i k}$.

The constitutive relationships in the linear elastic homogenized continuum are given as

$$
\sigma_{i j}^{e f f}(\mathbf{x})=c_{i j k l}^{e f f} \varepsilon_{k l}^{e f f}(\mathbf{x}),
$$

where $c_{i j k l}^{e f f}=$ const . Hence,

$$
\left\langle\sigma_{i j}^{e f f}\right\rangle_{B}=c_{i j k l}^{e f f}\left\langle\varepsilon_{k l}^{e f f}\right\rangle_{B}
$$

and from the symmetry the stress and strain tensors, we have the following symmetry properties

$$
c_{i j k l}^{e f f}=c_{j i k l}^{e f f}=c_{i j l k}^{e f f}
$$

while the symmetry

$$
c_{i j k l}^{e f f}=c_{k l i j}^{e f f}
$$

results from the requirement of linear elasticity. In view of Eqs. (9) and (10), we could calculate the effective material coefficients $c_{i j k l}^{e f f}$ if we knew either $\left[\sigma_{i j}^{e f f}(\mathbf{x}), \varepsilon_{k l}^{e f f}(\mathbf{x})\right]$ at a point $\mathbf{x}$ or the mean values $\left[\left\langle\sigma_{i j}^{e f f}\right\rangle_{B},\left\langle\varepsilon_{k l}^{e f f}\right\rangle_{B}\right]$. This would lead to an inverse problem. In order to eliminate the solution of the inverse problem, we try to identify the mean values $\left[\left\langle\sigma_{i j}^{e f f}\right\rangle_{B},\left\langle\varepsilon_{k l}^{e f f}\right\rangle_{B}\right]$ with the some quantities obtained from the solution of $m s-b v p$ in the RVE. 
If we consider a micro-structural KUBC in the RVE, it can be found (using the Green's function) that

$$
\varepsilon_{i j}^{m s}(\mathbf{x})=A_{i j k l}(\mathbf{x}) \bar{\varepsilon}_{k l} \quad \text { in } B,
$$

where $A_{i j k l}(\mathbf{x})$ is the influence tensor function corresponding to the KUBC. According to (4) and (7), we have $\left\langle\varepsilon_{i j}^{m s}(\mathbf{x})\right\rangle_{\Omega}=\bar{\varepsilon}_{i j}$. Hence and from (13)

$$
\left\langle A_{i j k l}(\mathbf{x})\right\rangle_{\Omega}=\delta_{i k} \delta_{j l} \text {. }
$$

Bearing in mind the constitutive law in the homogeneous skeleton $\Omega$

$$
\sigma_{i j}^{m s}(\mathbf{x})=c_{i j k l}^{s} \varepsilon_{k l}^{m s}(\mathbf{x})
$$

and $\sigma_{i j}^{m s}(\mathbf{x})=0$ in $(B-\Omega)$, we obtain from (13) and (15)

$$
\frac{|\Omega|}{|B|}\left\langle\sigma_{i j}^{m s}(\mathbf{x})\right\rangle_{\Omega}=\left\langle\sigma_{i j}^{m s}(\mathbf{x})\right\rangle_{B}=c_{i j k l}\left\langle A_{k l r s}(\mathbf{x})\right\rangle_{B} \bar{\varepsilon}_{r s} .
$$

Since $\bar{\varepsilon}_{r s}=\left\langle\varepsilon_{r s}^{m s}(\mathbf{x})\right\rangle_{\Omega}$, we can rewrite (16) as

$$
\left\langle\sigma_{i j}^{m s}(\mathbf{x})\right\rangle_{\Omega}=\frac{1}{1-p} c_{i j k l}^{s}\left\langle A_{k l r s}(\mathbf{x})\right\rangle_{B}\left\langle\varepsilon_{r s}^{m s}(\mathbf{x})\right\rangle_{\Omega}
$$

where we have introduced the porosity $p$ defined as

$$
p:=\frac{|B|-|\Omega|}{|B|}=1-\frac{|\Omega|}{|B|} .
$$

If we adopted (as physically admissible) identification $\left[\left\langle\sigma_{i j}^{e f f}\right\rangle_{B},\left\langle\varepsilon_{k l}^{e f f}\right\rangle_{B}\right]=$ $\left[\left\langle\sigma_{i j}^{m s}\right\rangle_{\Omega},\left\langle\varepsilon_{k l}^{m s}\right\rangle_{\Omega}\right]$, we would have form (17)

$$
\left\langle\sigma_{i j}^{e f f}\right\rangle_{B}=\frac{1}{1-p} c_{i j k l}^{s}\left\langle A_{k l r s}(\mathbf{x})\right\rangle_{B}\left\langle\varepsilon_{r s}^{e f f}\right\rangle_{B}
$$

and finally, in view of (10), we would have

$$
c_{i j r s}^{e f f}=\frac{1}{1-p} c_{i j k l}^{s}\left\langle A_{k l r s}(\mathbf{x})\right\rangle_{B} .
$$

Similarly, if we consider a micro-structural SUBC in the RVE, it can be found that

$$
\sigma_{i j}^{m s}(\mathbf{x})=B_{i j k l}(\mathbf{x}) \bar{\sigma}_{k l} \quad \text { in } B,
$$

where $B_{i j k l}(\mathbf{x})$ is the influence tensor function corresponding to the SUBC. According to (3) and (8), we have $\left\langle\sigma_{i j}^{m s}(\mathbf{x})\right\rangle_{\Omega}=\bar{\sigma}_{i j}$. Hence and from (21)

$$
\left\langle B_{i j k l}(\mathbf{x})\right\rangle_{\Omega}=\delta_{i k} \delta_{j l} \text {. }
$$

Bearing in mind the constitutive law in the homogeneous skeleton $\Omega$

$$
\varepsilon_{i j}^{m s}(\mathbf{x})=M_{i j k l}^{s} \sigma_{k l}^{m s}(\mathbf{x})
$$

(where $M_{i j k l}^{s}$ is the tensor of skeleton compliances) and $\varepsilon_{i j}^{m s}(\mathbf{x})=0$ in $(B-\Omega)$, we obtain from (21) and (23)

$$
\frac{|\Omega|}{|B|}\left\langle\varepsilon_{i j}^{m s}(\mathbf{x})\right\rangle_{\Omega}=\left\langle\varepsilon_{i j}^{m s}(\mathbf{x})\right\rangle_{B}=M_{i j k l}^{s}\left\langle B_{k l r s}(\mathbf{x})\right\rangle_{B} \bar{\sigma}_{r s} .
$$


Since $\bar{\sigma}_{r s}=\left\langle\sigma_{r s}^{m s}(\mathbf{x})\right\rangle_{\Omega}$, we can rewrite (24) as

$$
\left\langle\varepsilon_{i j}^{m s}(\mathbf{x})\right\rangle_{\Omega}=\frac{1}{1-p} M_{i j k l}^{s}\left\langle B_{k l r s}(\mathbf{x})\right\rangle_{B}\left\langle\sigma_{r s}^{m s}(\mathbf{x})\right\rangle_{\Omega}
$$

If we adopted (as physically admissible) identification $\left[\left\langle\sigma_{i j}^{e f f}\right\rangle_{B},\left\langle\varepsilon_{k l}^{e f f}\right\rangle_{B}\right]=$ $\left[\left\langle\sigma_{i j}^{m s}\right\rangle_{\Omega},\left\langle\varepsilon_{k l}^{m s}\right\rangle_{\Omega}\right]$, we would have form (25)

$$
\left\langle\varepsilon_{i j}^{e f f}(\mathbf{x})\right\rangle_{B}=\frac{1}{1-p} M_{i j k l}^{s}\left\langle B_{k l r s}(\mathbf{x})\right\rangle_{B}\left\langle\sigma_{r s}^{e f f}(\mathbf{x})\right\rangle_{B}
$$

and finally in view of $\left\langle\varepsilon_{i j}^{e f f}\right\rangle_{B}=M_{i j k l}^{e f f}\left\langle\sigma_{k l}^{e f f}\right\rangle_{B}$, we would have

$$
M_{i j r s}^{e f f}=\frac{1}{1-p} M_{i j k l}^{s}\left\langle B_{k l r s}(\mathbf{x})\right\rangle_{B} .
$$

Finding of the influence functions is not as simple task, in general. In what follows, we shall consider a special case of elliptical pores in 2D elasticity problems, when the analytical solution can be utilized. Another considered case is the numerical approach, which is applicable to empty pores of arbitrary shape and randomly distributed in the RVE. We shall illustrate this approach on elliptical pores uniformly distributed in the body.

\subsection{Analytical approaches}

It is well known that the analytical solution is available for elasticity problem in infinite plane with an elliptical empty void and applied tension load $\sigma_{i j}^{\infty}=\sigma \delta_{i 1} \delta_{j 1}$ at infinity [8-10]. Denoting the major and minor semi-axes as $a, b$ and selecting the RVE as square $l \times l$ with $l \gg a$, we can consider the analytical solution in infinite plane as certain approximation for the micro-structural boundary value problem in the RVE with prescribed constant traction load on the outer boundary and traction free boundary of the elliptical hole.

2

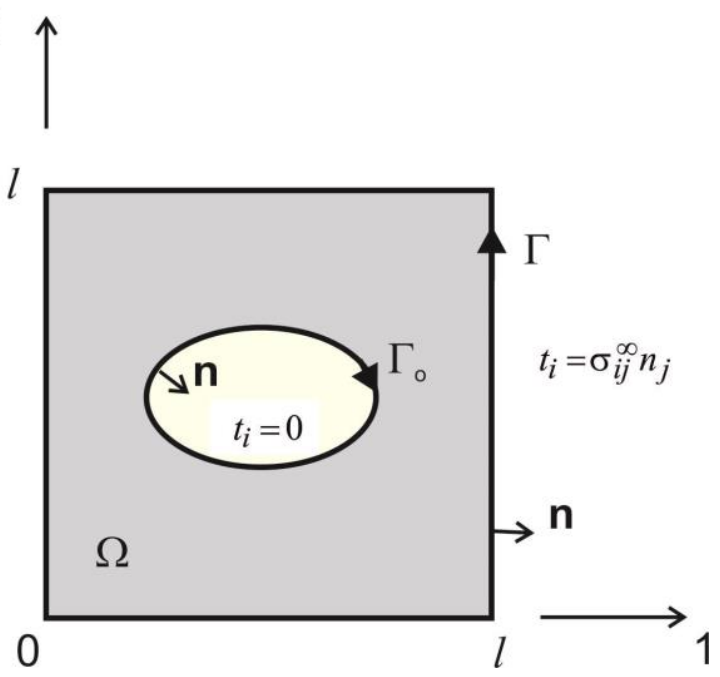

Fig. 1 Geometry of the quadrilateral RVE with one empty elliptical pore

Now, in view of Fig. 1, we can write

$$
\left\langle\sigma_{i j}^{m s}\right\rangle_{\Omega}:=\frac{1}{|\Omega|} \int_{\Omega} \sigma_{i j}^{m s}(\mathbf{x}) d \Omega=\frac{1}{2|\Omega|} \int_{\Omega}\left(\sigma_{i k}^{m s} x_{j, k}+\sigma_{j k}^{m s} x_{i, k}\right) d \Omega=
$$




$$
\begin{aligned}
& =\frac{1}{2|\Omega|} \int_{\Omega}\left[\left(\sigma_{i k}^{m s} x_{j}\right)_{, k}+\left(\sigma_{j k}^{m s} x_{i}\right)_{, k}\right] d \Omega=\frac{1}{2|\Omega|} \int_{\Gamma \cup \Gamma_{0}}\left(t_{i}^{m s} x_{j}+t_{j}^{m s} x_{i}\right) d \Gamma= \\
& =\frac{1}{2|\Omega|} \int_{\Gamma}\left(t_{i}^{m s} x_{j}+t_{j}^{m s} x_{i}\right) d \Gamma=\frac{|B|}{|\Omega|} \sigma_{i j}^{\infty}=\frac{1}{1-p} \sigma_{i j}^{\infty} \\
\left\langle\varepsilon_{i j}^{m s}\right\rangle_{\Omega} & :=\frac{1}{|\Omega|} \int_{\Omega} \varepsilon_{i j}^{m s}(\mathbf{x}) d \Omega=\frac{1}{2|\Omega|} \int_{\Omega}\left(u_{i, j}^{m s}+u_{j, i}^{m s}\right) d \Omega=\frac{1}{2|\Omega|} \int_{\Gamma \cup \Gamma_{0}}\left(u_{i}^{m s} n_{j}+u_{j}^{m s} n_{i}\right) d \Gamma= \\
& =\left\langle\varepsilon_{i j}^{m s}\right\rangle_{\Omega, \Gamma}+\left\langle\varepsilon_{i j}^{m s}\right\rangle_{\Omega, \Gamma_{0}}
\end{aligned}
$$

$$
\text { with }\left\langle\varepsilon_{i j}^{m s}\right\rangle_{\Omega, \Gamma}:=\frac{1}{2|\Omega|} \int_{\Gamma}\left(u_{i}^{m s} n_{j}+u_{j}^{m s} n_{i}\right) d \Gamma, \quad\left\langle\varepsilon_{i j}^{m s}\right\rangle_{\Omega, \Gamma_{0}}:=\frac{1}{2|\Omega|} \int_{\Gamma_{0}}\left(u_{i}^{m s} n_{j}+u_{j}^{m s} n_{i}\right) d \Gamma \text {. }
$$

Both these contributions to the mean value of micro-structural strains can be evaluated by using the exact solution for displacements in infinite plane. Apparently, in the linear theory of elasticity, both these integrals are to proportional to $\sigma_{i j}^{\infty}$, i.e.

$$
\left\langle\varepsilon_{i j}^{m s}\right\rangle_{\Omega, \Gamma}=\frac{1}{1-p} M_{i j k l} \sigma_{k l}^{\infty}, \quad\left\langle\varepsilon_{i j}^{m s}\right\rangle_{\Omega, \Gamma_{0}}=\frac{1}{1-p} H_{i j k l} \sigma_{k l}^{\infty},
$$

where $M_{i j k l} \sigma_{k l}^{\infty}:=\frac{1}{2|B|} \int_{\Gamma}\left(u_{i}^{m s} n_{j}+u_{j}^{m s} n_{i}\right) d \Gamma, H_{i j k l} \sigma_{k l}^{\infty}:=\frac{1}{2|B|} \int_{\Gamma_{0}}\left(u_{i}^{m s} n_{j}+u_{j}^{m s} n_{i}\right) d \Gamma$.

What we need is to find certain physically meaningful correlation between the mean values of stresses and strains in effective medium and those found from solution of micro-structural bvp. First, consider the integral force compatibility and average strain compatibility (IF\&ASC):

$$
\begin{aligned}
& \text { IFC: }|B|\left\langle\sigma_{i j}^{e f f}\right\rangle_{B}=|\Omega|\left\langle\sigma_{i j}^{m s}\right\rangle_{\Omega} \Rightarrow\left\langle\sigma_{i j}^{e f f}\right\rangle_{B}=(1-p)\left\langle\sigma_{i j}^{m s}\right\rangle_{\Omega}=\sigma_{i j}^{\infty} \\
& \text { ASC: }\left\langle\varepsilon_{i j}^{e f f}\right\rangle_{B}=\left\langle\varepsilon_{i j}^{m s}\right\rangle_{\Omega}=\frac{1}{1-p}\left(M_{i j k l}+H_{i j k l}\right) \sigma_{k l}^{\infty},
\end{aligned}
$$

where we have utilized Eqs. (28)-(31). Since $\left\langle\varepsilon_{i j}^{e f f}\right\rangle_{B}=M_{i j k l}^{e f f}\left\langle\sigma_{i j}^{e f f}\right\rangle_{B}$, we obtain from (32)-(33)

$$
M_{i j k l}^{e f f}=\frac{1}{1-p}\left(M_{i j k l}+H_{i j k l}\right)
$$

If we shifted the outer boundary of the RVE to infinity, i.e. $\Gamma \rightarrow \Gamma^{\infty}$, then $M_{i j k l} \rightarrow M_{i j k l}^{s}$, where $M_{i j k l}^{S}$ is the tensor of compliances corresponding to the matrix material of the skeleton. In view of such an approximation, we shall distinguish two analytical approaches within the IF\&ASC concept:

(i) analytical approach $I$, denoted as $\mathbf{A A}(\mathbf{I}): M_{i j k l}^{e f f}=\frac{1}{1-p}\left(M_{i j k l}^{s}+H_{i j k l}\right)$

(ii) analytical approach II, denoted as AA(II): $M_{i j k l}^{e f f}=\frac{1}{1-p}\left(M_{i j k l}+H_{i j k l}\right)$ 
Another concept consists in consideration of integral force compatibility and integral energy compatibility (IF\&EC):

IEC: $\left\langle\sigma_{i j}^{e f f}\right\rangle_{B}\left\langle\varepsilon_{i j}^{e f f}\right\rangle_{B}=\left\langle\sigma_{i j}^{m s}\right\rangle_{\Omega}\left\langle\varepsilon_{i j}^{m s}\right\rangle_{\Omega}$

hence, in view of (32), we have

$$
\left\langle\varepsilon_{i j}^{e f f}\right\rangle_{B}=\frac{1}{1-p}\left\langle\varepsilon_{i j}^{m s}\right\rangle_{\Omega}
$$

and finally in view of (29)-(31) and IFC (Eq.(32))

$$
\left\langle\varepsilon_{i j}^{e f f}\right\rangle_{B}=\frac{1}{1-p}\left\langle\varepsilon_{i j}^{m s}\right\rangle_{\Omega}=\frac{1}{(1-p)^{2}}\left(M_{i j k l}+H_{i j k l}\right) \sigma_{k l}^{\infty}=\frac{1}{(1-p)^{2}}\left(M_{i j k l}+H_{i j k l}\right)\left\langle\sigma_{i j}^{e f f}\right\rangle_{B} .
$$

Similar to the previous case, we shall distinguish other two analytical approaches:

(i) analytical approach III, denoted as AA(III): $M_{i j k l}^{e f f}=\frac{1}{(1-p)^{2}}\left(M_{i j k l}^{s}+H_{i j k l}\right)$

(ii) analytical approach IV, denoted as AA(IV): $M_{i j k l}^{e f f}=\frac{1}{(1-p)^{2}}\left(M_{i j k l}+H_{i j k l}\right)$

In order to evaluate the integrals $M_{i j k l}$ and $H_{i j k l}$, we employ the exact values for displacements found from the analytical solution in infinite plane (see Fig. 2)

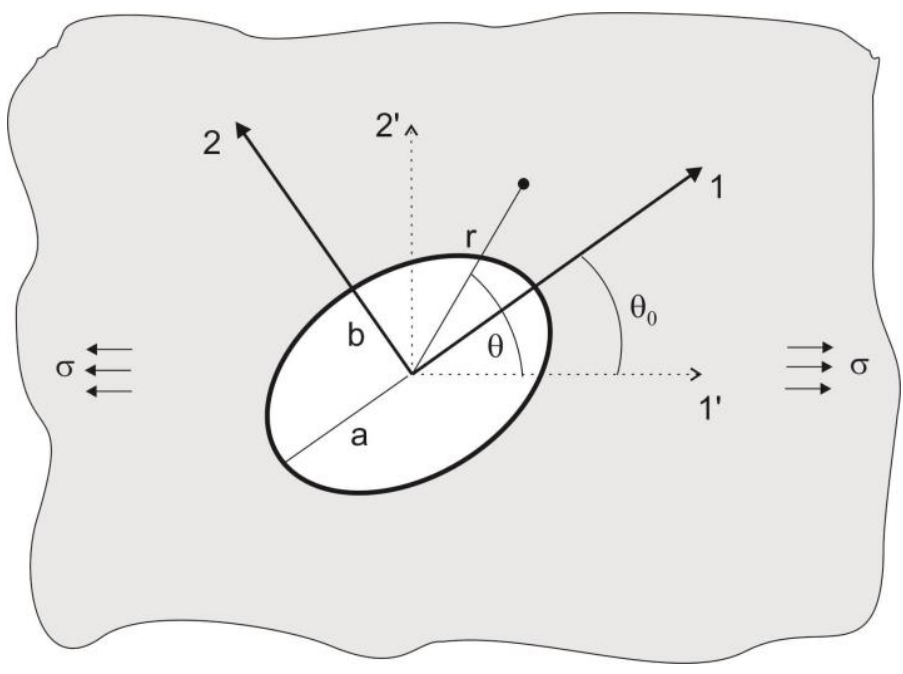

Fig. 2 Elliptical hole in infinite plane with applied uniform stress loading in infinity

$$
\begin{aligned}
u_{1}^{m s}(\rho, \theta) \approx & u_{1}(\rho, \theta)=\frac{\sigma R}{4} \frac{1+v}{E_{0}}\left\{\cos \theta\left[\Lambda\left(\rho-\frac{2 m}{\rho}\right)+\frac{1}{A}\left(2 \frac{1+m^{2}}{\rho}-\rho-2 m \frac{1+m^{2}}{\rho^{3}}+2 \frac{m^{3}}{\rho^{5}}\right)\right]+\right. \\
+ & \cos \left(\theta+2 \theta_{0}\right)\left[\frac{2 \Lambda}{\rho}+2 \rho+\frac{2}{m \rho}-\frac{1+m^{2}}{A} \frac{2}{m \rho}\right]+\cos \left(\theta-2 \theta_{0}\right) \frac{2}{A \rho^{3}}\left(1+m^{2}-\frac{m^{2}}{\rho^{2}}\right)+ \\
& \left.+\cos 3 \theta \frac{m}{A \rho}\left(\frac{m}{\rho^{2}}-2\right)+\cos \left(3 \theta+2 \theta_{0}\right) \frac{2}{A \rho}\right\}
\end{aligned}
$$




$$
\begin{aligned}
u_{2}^{m s}(\rho, \theta) \approx & u_{2}(\rho, \theta)=\frac{\sigma R}{4} \frac{1+v}{E_{0}}\left\{\sin \theta\left[\Lambda\left(\rho+\frac{2 m}{\rho}\right)+\frac{1}{A}\left(2 \frac{1+m^{2}}{\rho}-\rho+2 m \frac{1+m^{2}}{\rho^{3}}-2 \frac{m^{3}}{\rho^{5}}\right)\right]+\right. \\
+ & \sin \left(\theta+2 \theta_{0}\right)\left[\frac{2}{m \rho}-\frac{2 \Lambda}{\rho}-2 \rho-\frac{2}{A} \frac{1+m^{2}}{m \rho}\right]-\sin \left(\theta-2 \theta_{0}\right) \frac{2}{A \rho^{3}}\left(1+m^{2}-\frac{m^{2}}{\rho^{2}}\right)- \\
& \left.-\sin 3 \theta \frac{m}{A \rho}\left(2+\frac{m}{\rho^{2}}\right)+\sin \left(3 \theta+2 \theta_{0}\right) \frac{2}{A \rho}\right\}
\end{aligned}
$$

Performing the required integrations, we obtain

$$
\left(\begin{array}{c}
H_{11 k l} \sigma_{k l}^{\infty} \\
H_{22 k l} \sigma_{k l}^{\infty} \\
H_{12 k l} \sigma_{k l}^{\infty}
\end{array}\right)=\left(\begin{array}{lll}
H_{1111} & H_{1122} & 2 H_{1112} \\
H_{2211} & H_{2222} & 2 H_{2212} \\
H_{1211} & H_{1222} & 2 H_{1212}
\end{array}\right)\left(\begin{array}{c}
\sigma_{11}^{\infty} \\
\sigma_{22}^{\infty} \\
\sigma_{12}^{\infty}
\end{array}\right),[H]=p \frac{1+v}{E_{0}}\left(\begin{array}{ccc}
B_{1} & B_{2} & 0 \\
B_{3} & B_{4} & 0 \\
0 & 0 & B_{5}
\end{array}\right)
$$

in which

$$
\begin{aligned}
& H_{1111}=\frac{\pi R b}{4|B|} \frac{1+v}{E_{0}}\left[\Lambda(3-2 m)+3-m-m^{2}\right]=p \frac{1+v}{E_{0}} B_{1}, \quad B_{1}:=\frac{1+\gamma}{8}\left[\Lambda(3-2 m)+3-m-m^{2}\right] \\
& H_{1122}=\frac{\pi R b}{4|B|} \frac{1+v}{E_{0}}\left[-\Lambda(1+2 m)-\left(1+m+m^{2}\right)\right]=p \frac{1+v}{E_{0}} B_{2}, \quad B_{2}:=\frac{1+\gamma}{8}\left[-\Lambda(1+2 m)-\left(1+m+m^{2}\right)\right] \\
& H_{2211}=\frac{\pi R a}{4|B|} \frac{1+v}{E_{0}}\left[\Lambda(2 m-1)-1+m-m^{2}\right]=p \frac{1+v}{E_{0}} B_{3}, \quad B_{3}:=\frac{1+\gamma}{8 \gamma}\left[\Lambda(2 m-1)-1+m-m^{2}\right] \\
& H_{2222}=\frac{\pi R a}{4|B|} \frac{1+v}{E_{0}}\left[\Lambda(3+2 m)+3+m-m^{2}\right]=p \frac{1+v}{E_{0}} B_{4}, \quad B_{4}:=\frac{1+\gamma}{8 \gamma}\left[\Lambda(3+2 m)+3+m-m^{2}\right] \\
& 2 H_{1212}=\frac{\pi R(a+b)}{2|B|} \frac{1+v}{E_{0}}(\Lambda+1)=p \frac{1+v}{E_{0}} B_{5} \quad, \quad B_{5}:=\frac{1}{2}\left[1+\frac{1}{2}\left(\gamma+\frac{1}{\gamma}\right)\right](\Lambda+1)
\end{aligned}
$$

with

$$
p:=\frac{\pi a b}{l^{2}}, \quad m=\frac{a-b}{a+b}=\frac{1-\gamma}{1+\gamma}, \quad \gamma:=\frac{b}{a}, \quad \Lambda=\frac{\lambda+3 \mu}{\lambda+\mu}=3-4 \tilde{v},
$$

where $\lambda$ and $\mu$, are Lame coefficients which are correlated with the Young modulus $E_{0}$ and Poisson ratio $v$ by

$\mu=\frac{E_{0}}{2(1+v)}, \quad \lambda=\mu \frac{2 \tilde{v}}{1-2 \tilde{v}}, \quad \tilde{v}=\left\{\begin{array}{cc}v /(1+v), & \text { for plane stress problems } \\ v, & \text { otherwise }\end{array}\right.$

The matrix of compliances of the homogeneous, isotropic and linear elastic skeleton is given as

$$
\left[M^{s}\right]=\left(\begin{array}{lll}
M_{1111}^{s} & M_{1122}^{s} & 2 M_{1112}^{s} \\
M_{2211}^{s} & M_{2222}^{s} & 2 M_{2212}^{s} \\
M_{1211}^{s} & M_{1222}^{s} & 2 M_{1212}^{s}
\end{array}\right)=\frac{1+v}{4 E_{0}}\left(\begin{array}{ccc}
\Lambda+1 & \Lambda-3 & 0 \\
\Lambda-3 & \Lambda+1 & 0 \\
0 & 0 & 4
\end{array}\right) .
$$


Note that in the case of elliptic void, we have not the closed form expression for the integrals in the matrix $M_{i j k l}$, though we have it in the case of circular void

$$
\begin{aligned}
& M_{1111}=M_{1111}^{s}+\frac{1+v}{2 E_{0}}\left[(\Lambda \pi+4) \frac{p}{\pi}+2(\pi+2)\left(\frac{p}{\pi}\right)^{2}\right], \quad M_{1112}=0, \\
& M_{1122}=M_{2211}=M_{1122}^{s}-\frac{1+v}{4 E_{0}}\left[((\Lambda-2) \pi+4) \frac{p}{\pi}-2(\pi+2)\left(\frac{p}{\pi}\right)^{2}\right], \quad M_{2212}=0, \\
& 2 M_{1212}=2 M_{1212}^{s}+\frac{1+v}{E_{0}}\left[\Lambda p-2(\pi+2)\left(\frac{p}{\pi}\right)^{2}\right], \quad M_{1211}=0=M_{1222}
\end{aligned}
$$

Performing the limit $b \rightarrow a$, when $m \rightarrow 0$ and $\gamma \rightarrow 1$ in Eq. (43), we obtain the $H_{i j k l}$ matrix for circular pore

$$
\begin{aligned}
& B_{1} \rightarrow 3 \frac{\Lambda+1}{4} \text { and } H_{1111}=3 \frac{Q}{E_{0}}, \quad B_{4} \rightarrow 3 \frac{\Lambda+1}{4} \text { and } H_{2222}=3 \frac{Q}{E_{0}}, \quad Q=p \frac{\Lambda+1}{4}(1+v) \\
& B_{2} \rightarrow-\frac{\Lambda+1}{4} \text { and } H_{1122}=-\frac{Q}{E_{0}}, \quad B_{3} \rightarrow-\frac{\Lambda+1}{4} \text { and } H_{2211}=-\frac{Q}{E_{0}} \\
& B_{5} \rightarrow \Lambda+1 \text { and } 2 H_{1212}=4 \frac{Q}{E_{0}} .
\end{aligned}
$$

Thus, in the case of circular pore, the effective continuum is isotropic, while in the case of oriented elliptical pore it is anisotropic (it is neither isotropic nor orthotropic, since $B_{2} \neq B_{3}$ ). However, if we consider elliptical pore randomly oriented, we obtain the compliance matrix by angular averaging of the tensor of compliances for elliptical pores with sloped major axis with respect to the applied loading. Making use the transformation properties of tensors,

$$
\left\langle M_{i j k l}^{e f f}\right\rangle_{\psi}:=\frac{1}{2 \pi} \int_{0}^{2 \pi} \tilde{M}_{i j k l}^{e f f} d \psi, \quad \tilde{M}_{i j k l}^{e f f}=\mathrm{O}_{i \alpha}^{T}(\psi) \mathrm{O}_{j \beta}^{T}(\psi) \mathrm{O}_{k \gamma}^{T}(\psi) \mathrm{O}_{l \delta}^{T}(\psi) M_{i j k l}^{e f f}
$$

one can perform the angular averaging in closed form

$$
\begin{aligned}
& \left\langle M_{1111}^{\text {eff }}\right\rangle_{\psi}=\frac{3}{8}\left(M_{1111}^{\text {eff }}+M_{2222}^{\text {eff }}\right)+\frac{1}{8}\left(M_{1122}^{\text {eff }}+M_{2211}^{\text {eff }}+4 M_{1212}^{\text {eff }}\right)=\left\langle M_{2222}^{\text {eff }}\right\rangle_{\psi} \\
& \left\langle M_{1122}^{\text {eff }}\right\rangle_{\psi}=\frac{1}{8}\left(M_{1111}^{\text {eff }}+M_{2222}^{\text {eff }}-4 M_{1212}^{\text {eff }}\right)+\frac{3}{8}\left(M_{1122}^{\text {eff }}+M_{2211}^{\text {eff }}\right)=\left\langle M_{2211}^{\text {eff }}\right\rangle_{\psi} \\
& \left\langle M_{1112}^{\text {eff }}\right\rangle_{\psi}=0=\left\langle M_{2212}^{\text {eff }}\right\rangle_{\psi}=\left\langle M_{1211}^{\text {eff }}\right\rangle_{\psi}=\left\langle M_{1222}^{\text {eff }}\right\rangle_{\psi} \\
& \left\langle M_{1212}^{\text {eff }}\right\rangle_{\psi}=\frac{1}{8}\left(M_{1111}^{\text {eff }}+M_{2222}^{\text {eff }}-M_{1122}^{\text {eff }}-M_{2211}^{\text {eff }}\right)+\frac{1}{2} M_{1212}^{\text {eff }} .
\end{aligned}
$$

Thus, the effective continuum in the case of randomly oriented elliptical pore is isotropic.

Having known the matrices $M_{i j k l}, M_{i j k l}^{s}, H_{i j k l}$ one can find the matrices of compliances for effective continuum in particular approaches AA(I), AA(II), AA(III) and AA(IV). Recall that the matrix $M_{i j k l}$ is known in closed form only for circular pore, but not for elliptical pore. Thus, for the elliptical pore, we have closed form results only by AA(I) and AA(III). The ma- 
trix of stiffness coefficients for effective continuum is obtained by inversion of the matrix of compliances.

\subsection{Numerical approach}

If the shape of pore is arbitrary, one can solve the micro-structural bvp in the RVE numerically and get the micro-structural mean values of strains and stresses. If we consider the boundary of the pore to be traction-free $\left.t_{i}\right|_{\Gamma_{0}}=0$, we can express the mean values in terms of boundary integrals as

$$
\begin{aligned}
\left\langle\sigma_{i j}^{m s}\right\rangle_{\Omega} & :=\frac{1}{|\Omega|} \int_{\Omega} \sigma_{i j}^{m s}(\mathbf{x}) d \Omega=\frac{1}{2|\Omega|} \int_{\Omega}\left(\sigma_{i k}^{m s} x_{j, k}+\sigma_{j k}^{m s} x_{i, k}\right) d \Omega= \\
& =\frac{1}{2|\Omega|} \int_{\Omega}\left[\left(\sigma_{i k}^{m s} x_{j}\right)_{, k}+\left(\sigma_{j k}^{m s} x_{i}\right)_{, k}\right] d \Omega=\frac{1}{2|\Omega|} \int_{\Gamma \cup \Gamma_{0}}\left(t_{i}^{m s} x_{j}+t_{j}^{m s} x_{i}\right) d \Gamma= \\
& =\frac{1}{2|\Omega|} \int_{\Gamma}\left(t_{i}^{m s} x_{j}+t_{j}^{m s} x_{i}\right) d \Gamma \\
\left\langle\varepsilon_{i j}^{m s}\right\rangle_{\Omega} & :=\frac{1}{|\Omega|} \int_{\Omega} \varepsilon_{i j}^{m s}(\mathbf{x}) d \Omega=\frac{1}{2|\Omega|} \int_{\Omega}\left(u_{i, j}^{m s}+u_{j, i}^{m s}\right) d \Omega=\frac{1}{2|\Omega|} \int_{\Gamma \cup \Gamma_{0}}\left(u_{i}^{m s} n_{j}+u_{j}^{m s} n_{i}\right) d \Gamma= \\
& =\left\langle\varepsilon_{i j}^{m s}\right\rangle_{\Omega, \Gamma}+\left\langle\varepsilon_{i j}^{m s}\right\rangle_{\Omega, \Gamma_{0}}
\end{aligned}
$$

with $\left\langle\varepsilon_{i j}^{m s}\right\rangle_{\Omega, \Gamma}:=\frac{1}{2|\Omega|} \int_{\Gamma}\left(u_{i}^{m s} n_{j}+u_{j}^{m s} n_{i}\right) d \Gamma$,

$$
\left\langle\varepsilon_{i j}^{m s}\right\rangle_{\Omega, \Gamma_{0}}:=\frac{1}{2|\Omega|} \int_{\Gamma_{0}}\left(u_{i}^{m s} n_{j}+u_{j}^{m s} n_{i}\right) d \Gamma=\frac{|B|}{|\Omega|} \Delta \varepsilon_{i j}^{m s}, \Delta \varepsilon_{i j}^{m s}:=\frac{1}{2|B|} \int_{\Gamma_{0}}\left(u_{i}^{m s} n_{j}+u_{j}^{m s} n_{i}\right) d \Gamma
$$

Since the mean values of the strains and stresses in effective continuum are not known (we cannot solve any direct bvp in effective medium because of absence of material coefficients), we should find a physically meaningful correlation between $\left[\left\langle\sigma_{i j}^{e f f}\right\rangle_{B},\left\langle\varepsilon_{k l}^{e f f}\right\rangle_{B}\right]$ and $\left[\left\langle\sigma_{i j}^{m s}\right\rangle_{\Omega},\left\langle\varepsilon_{k l}^{m s}\right\rangle_{\Omega}\right]$ in order to get the stiffness coefficients in the homogenized effective continuum

$$
\left(\begin{array}{l}
\sigma_{11}^{\text {eff }} \\
\sigma_{22}^{\text {eff }} \\
\sigma_{12}^{\text {eff }}
\end{array}\right)=\left(\begin{array}{ccc}
c_{11}^{\text {eff }} & c_{12}^{\text {eff }} & c_{16}^{\text {eff }} \\
c_{21}^{\text {eff }} & c_{22}^{\text {eff }} & c_{26}^{\text {eff }} \\
c_{61}^{\text {eff }} & c_{62}^{\text {eff }} & c_{66}^{\text {eff }}
\end{array}\right)\left(\begin{array}{c}
\varepsilon_{11}^{\text {eff }} \\
\varepsilon_{22}^{\text {eff }} \\
2 \varepsilon_{12}^{\text {eff }}
\end{array}\right) \text { or }\left(\begin{array}{l}
\left\langle\sigma_{11}^{\text {eff }}\right\rangle_{B} \\
\left\langle\sigma_{22}^{\text {eff }}\right\rangle_{B} \\
\left\langle\sigma_{12}^{\text {eff }}\right\rangle_{B}
\end{array}\right)=\left(\begin{array}{ccc}
c_{11}^{\text {eff }} & c_{12}^{\text {eff }} & c_{16}^{\text {eff }} \\
c_{21}^{\text {eff }} & c_{22}^{\text {eff }} & c_{26}^{\text {eff }} \\
c_{61}^{\text {eff }} & c_{62}^{\text {eff }} & c_{66}^{\text {eff }}
\end{array}\right)\left(\begin{array}{c}
\left\langle\varepsilon_{11}^{\text {eff }}\right\rangle_{B} \\
\left\langle\varepsilon_{22}^{\text {eff }}\right\rangle_{B} \\
2\left\langle\varepsilon_{12}^{\text {eff }}\right\rangle_{B}
\end{array}\right) \text {. }
$$

For this purpose, we shall use two approaches:

(i) boundary densities compatibility (BDC) approach: we assume that the physically relevant boundary densities on the outer boundary of the RVE are the same for both the microstructural bvp and the bvp in effective continuum, i.e. $\left.u_{i}^{e f f}\right|_{\Gamma}=\left.u_{i}^{m s}\right|_{\Gamma},\left.t_{i}^{e f f}\right|_{\Gamma}=\left.t_{i}^{m s}\right|_{\Gamma}$. Then,

$$
\left\langle\varepsilon_{i j}^{e f f}\right\rangle_{B}:=\frac{1}{|B|} \int_{B} \varepsilon_{i j}^{e f f}(\mathbf{x}) d \Omega=\frac{1}{2|B|} \int_{B}\left(u_{i, j}^{e f f}+u_{j, i}^{e e f f}\right) d \Omega=\frac{1}{2|B|} \int_{\Gamma}\left(u_{i}^{e f f} n_{j}+u_{j}^{e f f} n_{i}\right) d \Gamma=
$$




$$
\begin{gathered}
=\frac{1}{2|B|} \int_{\Gamma}\left(u_{i}^{m s} n_{j}+u_{j}^{m s} n_{i}\right) d \Omega=(1-p)\left\langle\varepsilon_{i j}^{m s}\right\rangle_{\Omega, \Gamma}, \\
\left\langle\sigma_{i j}^{e f f}\right\rangle_{B}:=\frac{1}{|B|} \int_{B} \sigma_{i j}^{e f f}(\mathbf{x}) d \Omega=\frac{1}{2|B|} \int_{B}\left(\sigma_{i k}^{e f f} x_{j, k}+\sigma_{j k}^{e f f} x_{i, k}\right) d \Omega= \\
=\frac{1}{2|B|} \int_{B}\left[\left(\sigma_{i k}^{e f f} x_{j}\right)_{, k}+\left(\sigma_{j k}^{e f f} x_{i}\right)_{, k}\right] d \Omega=\frac{1}{2|B|} \int_{\Gamma}\left(t_{i}^{e f f} x_{j}+t_{j}^{e f f} x_{i}\right) d \Gamma= \\
=\frac{1}{2|B|} \int_{\Gamma}\left(t_{i}^{m s} x_{j}+t_{j}^{m s} x_{i}\right) d \Gamma=(1-p)\left\langle\sigma_{i j}^{m s}\right\rangle_{\Omega} .
\end{gathered}
$$

(ii) integral force \& energy compatibilities (IF\&EC) approach: we consider two assumptions:

(1) integral force equilibrium: $|B|\left\langle\sigma_{i j}^{e f f}\right\rangle_{B}=|\Omega|\left\langle\sigma_{i j}^{m s}\right\rangle_{\Omega}$

(2) energy equilibrium:

$$
\begin{aligned}
\left.\begin{array}{l}
\left\langle\sigma_{i j}^{e f f} \varepsilon_{i j}^{e f f}\right\rangle_{B}=\left\langle\sigma_{i j}^{m s} \varepsilon_{i j}^{m s}\right\rangle_{\Omega} \\
\left\langle\sigma_{i j}^{e f f} \varepsilon_{i j}^{e f f}\right\rangle_{B}=\left\langle\sigma_{i j}^{e f f}\right\rangle_{B}\left\langle\varepsilon_{i j}^{e f f}\right\rangle_{B}
\end{array}\right\} \Rightarrow \\
\Rightarrow\left\{\begin{array}{c}
\left\langle\sigma_{i j}^{e f f}\right\rangle_{B}=(1-p)\left\langle\sigma_{i j}^{m s}\right\rangle_{\Omega} \\
\left\langle\sigma_{i j}^{m s}\right\rangle_{\Omega}\left\langle\varepsilon_{i j}^{e f f}\right\rangle_{B}=\frac{1}{1-p}\left\langle\sigma_{i j}^{m s} \varepsilon_{i j}^{m s}\right\rangle_{\Omega}
\end{array}\right.
\end{aligned}
$$

Since

$$
\begin{aligned}
\left\langle\sigma_{i j}^{m s} \varepsilon_{i j}^{m s}\right\rangle_{\Omega}= & \frac{1}{|\Omega|} \int_{\partial \Omega} t_{i}^{m s} u_{i}^{m s} d \Gamma=\frac{1}{|\Omega|} \int_{\Gamma} t_{i}^{m s} u_{i}^{m s} d \Gamma \\
|\Omega|\left\langle\sigma_{i j}^{m s} \varepsilon_{i j}^{m s}\right\rangle_{\Omega} & \left.-\left\langle\sigma_{i j}^{m s}\right\rangle_{\Omega}\left\langle\varepsilon_{i j}^{m s}\right\rangle_{\Omega}\right)=\int_{\Gamma} t_{i}^{m s} u_{i}^{m s} d \Gamma-\left\langle\varepsilon_{i j}^{m s}\right\rangle_{\Omega} \int_{\Gamma} t_{i}^{m s} x_{j} d \Gamma- \\
& -|\Omega|\left\langle\sigma_{i k}^{m s}\right\rangle_{\Omega}\left\langle\varepsilon_{i k}^{m s}\right\rangle_{\Omega}+\left\langle\sigma_{i k}^{m s}\right\rangle_{\Omega}\left\langle\varepsilon_{i k}^{m s}\right\rangle_{\Omega} \int_{\Gamma+\Gamma_{0}} x_{j} n_{k} d \Gamma= \\
& =\int_{\Gamma}\left(t_{i}^{m s}-n_{k}\left\langle\sigma_{i k}^{m s}\right\rangle_{\Omega}\right)\left(u_{i}^{m s}-x_{j}\left\langle\varepsilon_{i j}^{m s}\right\rangle_{\Omega}\right) d \Gamma+ \\
& +\left\langle\sigma_{i j}^{m s}\right\rangle_{\Omega}\left\langle\varepsilon_{i k}^{m s}\right\rangle_{\Omega} \underbrace{\int_{\Gamma_{0}} x_{j} n_{k} d \Gamma}_{-(|B|-|\Omega|) \delta_{j k}}-\left\langle\sigma_{i k}^{m s}\right\rangle_{\Omega} \underbrace{\mid \varepsilon_{i k}^{m s}}_{|B|\left\langle\varepsilon_{i k}^{m s}\right\rangle_{\Omega, \Gamma_{0}}}
\end{aligned}
$$

we have

$$
\left\langle\sigma_{i j}^{m s} \varepsilon_{i j}^{m s}\right\rangle_{\Omega}=\frac{1}{1-p}\left\langle\sigma_{i j}^{m s}\right\rangle_{\Omega}\left((1-2 p)\left\langle\varepsilon_{i j}^{m s}\right\rangle_{\Omega}-\Delta \varepsilon_{i j}^{m s}\right)+\int_{\Gamma}\left(t_{i}^{m s}-n_{k}\left\langle\sigma_{i k}^{m s}\right\rangle_{\Omega}\right)\left(u_{i}^{m s}-x_{j}\left\langle\varepsilon_{i j}^{m s}\right\rangle_{\Omega}\right) d \Gamma
$$

The last integral is vanishing if the KUBC or SUBC are employed on the outer boundary of the RVE. Then, 


$$
\left\langle\varepsilon_{i j}^{e f f}\right\rangle_{B}=\frac{1}{(1-p)^{2}}\left((1-2 p)\left\langle\varepsilon_{i j}^{m s}\right\rangle_{\Omega}-\Delta \varepsilon_{i j}^{m s}\right)
$$

The evaluation of mean values of micro-structural fields is remarkably simplified, if we consider KUBC (kinematic uniform b.c.) on the outer boundary of the RVE, i.e. $\left.u_{i}^{m s}\right|_{\Gamma}=\tilde{u}_{i}$ with $\tilde{u}_{i}:=\left.\bar{\varepsilon}_{i k} x_{k}\right|_{\Gamma}, \bar{\varepsilon}_{i k}=$ const.

Then,

$$
\begin{gathered}
\left\langle\varepsilon_{i j}^{m s}\right\rangle_{\Omega, \Gamma}:=\frac{1}{2|\Omega|} \int_{\Gamma}\left(u_{i}^{m s} n_{j}+u_{j}^{m s} n_{i}\right) d \Gamma=\frac{1}{2|\Omega|} \int_{\Gamma}\left(\bar{\varepsilon}_{i k} x_{k} n_{j}+\bar{\varepsilon}_{j k} x_{k} n_{i}\right) d \Omega= \\
=\frac{1}{2|\Omega|} \int_{B}\left(\bar{\varepsilon}_{i k} x_{k, j}+\bar{\varepsilon}_{j k} x_{k, i}\right) d \Omega=\frac{|B|}{|\Omega|} \bar{\varepsilon}_{i j}=\frac{1}{1-p} \bar{\varepsilon}_{i j}
\end{gathered}
$$

Moreover, in the case of uniform distribution of pores in the macrostructure, we can create identical rectangular cells around each pore with periodic boundary conditions on the boundaries of cells. Then, if we create the RVE from several cells, we can see that because of the symmetry and consideration of rigid body motion equivalence, the solution in each cell is the same. Therefore it is sufficient to solve the micro-structural bvp in one cell with one pore in the case of uniform distribution of pores in the macrostructure. In what follows, we restrict our consideration to KUBC.

Thus, we can find the mean values of strains and stresses in the effective continuum as

(i) BDC-approach:

$$
\begin{aligned}
& \left\langle\varepsilon_{i j}^{e f f}\right\rangle_{B}=(1-p)\left\langle\varepsilon_{i j}^{m s}\right\rangle_{\Omega, \Gamma}=\bar{\varepsilon}_{i j} \\
& \left\langle\sigma_{i j}^{e f f}\right\rangle_{B}=(1-p)\left\langle\sigma_{i j}^{m s}\right\rangle_{\Omega}=\frac{1}{2|B|} \int_{\Gamma}\left(t_{i}^{m s} x_{j}+t_{j}^{m s} x_{i}\right) d \Gamma
\end{aligned}
$$

where $t_{i}^{m s}$ on $\Gamma$ should be got from the solution of the micro-structural bvp.

(ii) IF\&EC-approach:

$$
\begin{aligned}
\left\langle\varepsilon_{i j}^{e f f}\right\rangle_{B} & =\frac{1}{(1-p)^{2}}\left[(1-2 p)\left\langle\varepsilon_{i j}^{m s}\right\rangle_{\Omega}-\Delta \varepsilon_{i j}^{m s}\right]= \\
& =\frac{1}{(1-p)^{2}}\left[(1-2 p)\left(\left\langle\varepsilon_{i j}^{m s}\right\rangle_{\Omega, \Gamma}+\frac{1}{1-p} \Delta \varepsilon_{i j}^{m s}\right)-\Delta \varepsilon_{i j}^{m s}\right]= \\
& =\frac{1}{(1-p)^{3}}\left[(1-2 p) \bar{\varepsilon}_{i j}-p \Delta \varepsilon_{i j}^{m s}\right], \quad \Delta \varepsilon_{i j}^{m s}=\frac{1}{2|B|} \int_{\Gamma_{0}}\left(u_{i}^{m s} n_{j}+u_{j}^{m s} n_{i}\right) d \Gamma \\
\left\langle\sigma_{i j}^{e f f}\right\rangle_{B} & =(1-p)\left\langle\sigma_{i j}^{m s}\right\rangle_{\Omega}=\frac{1}{2|B|} \int_{\Gamma}\left(t_{i}^{m s} x_{j}+t_{j}^{m s} x_{i}\right) d \Gamma
\end{aligned}
$$

where the integrands in both the integrals over $\Gamma_{0}$ and $\Gamma$ are taken from the solution of the micro-structural bvp.

Now, we show that it is sufficient to solve three micro-structural bvp in order to get all material coefficients in homogenized effective elastic continuum: 
b.v.p. (I): $\quad u_{i}^{I}(\mathbf{x})=\left.\bar{\varepsilon}_{i k}^{I} x_{k}\right|_{\Gamma}, \quad \bar{\varepsilon}_{i k}^{I}=\bar{\varepsilon}_{11}^{I} \delta_{i 1} \delta_{k 1} ;\left.\quad t_{i}^{I}(\mathbf{x})\right|_{\Gamma_{0}}=0$

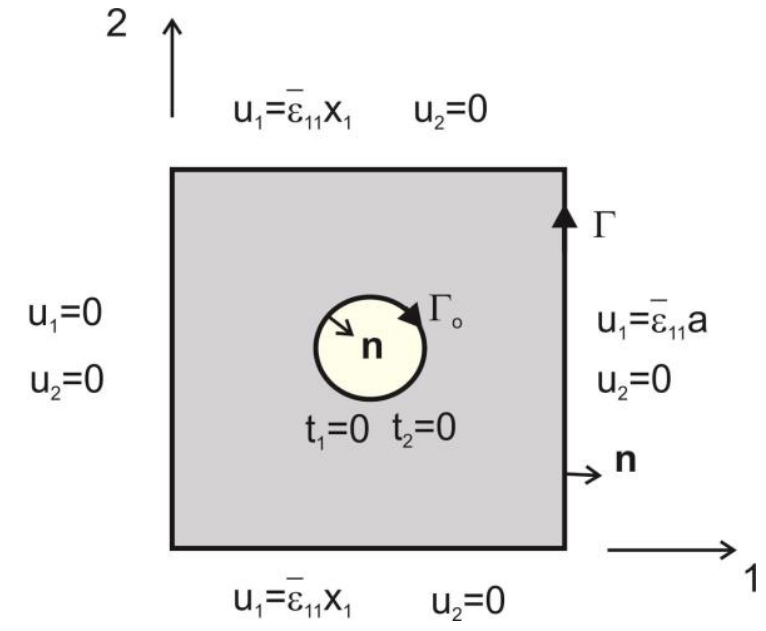

Fig. 3 The first choice of boundary conditions in quadrilateral RVE with one empty pore Having solved the considered $m s-b v p$, one can evaluate

$$
\left\langle\sigma_{i j}^{(I) e f f}\right\rangle_{B}=\frac{1}{2 l^{2}} \int_{\Gamma}\left(t_{i}^{(I) m s} x_{j}+t_{j}^{(I) m s} x_{i}\right) d \Gamma, \quad \Delta \varepsilon_{i j}^{(I) m s}=\frac{1}{2 l^{2}} \int_{\Gamma_{0}}\left(u_{i}^{(I) m s} n_{j}+u_{j}^{(I) m s} n_{i}\right) d \Gamma
$$

b.v.p. (II): $\quad \tilde{u}_{i}^{I I}(\mathbf{x})=\left.\bar{\varepsilon}_{i k}^{I I} x_{k}\right|_{\Gamma}, \quad \bar{\varepsilon}_{i k}^{I I}=\bar{\varepsilon}_{22}^{I I} \delta_{i 2} \delta_{k 2} ;\left.\quad t_{i}^{I I}(\mathbf{x})\right|_{\Gamma_{0}}=0$

2<smiles>CC</smiles>

$\mathrm{u}_{1}=0 \quad \mathrm{u}_{2}=\bar{\varepsilon}_{22} \mathrm{~b}$

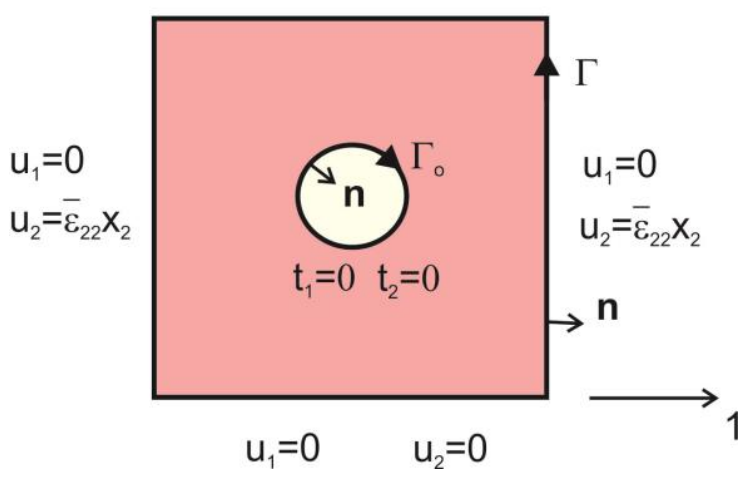

Fig. 4 The second choice of boundary conditions in quadrilateral RVE with one empty pore Having solved the considered $m s-b v p$, one can evaluate

$$
\left\langle\sigma_{i j}^{(I I) e f f}\right\rangle_{B}=\frac{1}{2 l^{2}} \int_{\Gamma}\left(t_{i}^{(I I) m s} x_{j}+t_{j}^{(I I) m s} x_{i}\right) d \Gamma, \quad \Delta \varepsilon_{i j}^{(I I) m s}=\frac{1}{2 l^{2}} \int_{\Gamma_{0}}\left(u_{i}^{(I I) m s} n_{j}+u_{j}^{(I I) m s} n_{i}\right) d \Gamma
$$

b.v.p. (III): $\tilde{u}_{i}^{I I I}(\mathbf{x})=\left.\bar{\varepsilon}_{i k}^{I I I} x_{k}\right|_{\Gamma}, \bar{\varepsilon}_{i k}^{I I I}=\bar{\varepsilon}_{12}^{I I I}\left(\delta_{i 1} \delta_{k 2}+\delta_{i 2} \delta_{k 1}\right) ;\left.\quad t_{i}^{I I I}(\mathbf{x})\right|_{\Gamma_{0}}=0$ 


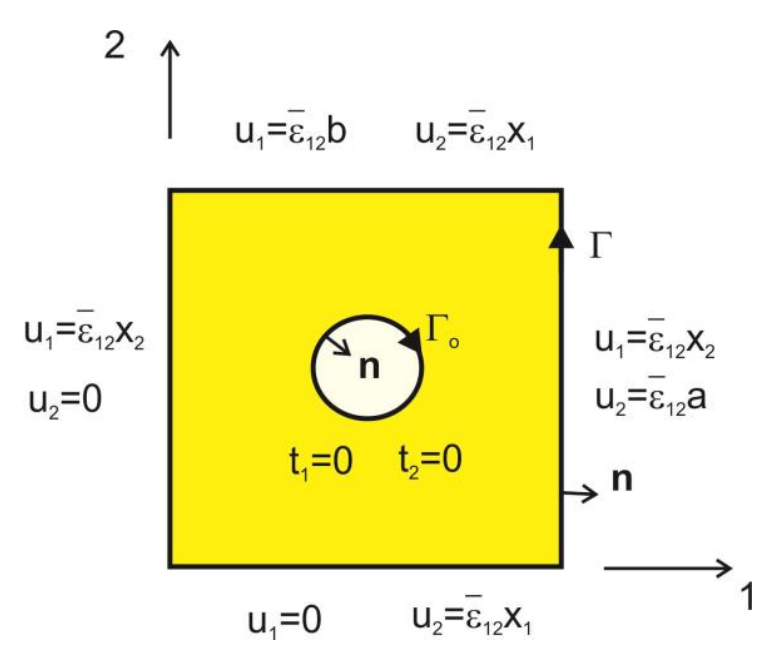

Fig. 5 The third choice of boundary conditions in quadrilateral RVE with one empty pore Having solved the considered $m s-b v p$, one can evaluate

$$
\left\langle\sigma_{i j}^{(I I I) e f f}\right\rangle_{B}=\frac{1}{2 l^{2}} \int_{\Gamma}\left(t_{i}^{(I I I) m s} x_{j}+t_{j}^{(I I I) m s} x_{i}\right) d \Gamma, \quad \Delta \varepsilon_{i j}^{(I I I) m s}=\frac{1}{2 l^{2}} \int_{\Gamma_{0}}\left(u_{i}^{(I I I) m s} n_{j}+u_{j}^{(I I I) m s} n_{i}\right) d \Gamma
$$

According to Eqs. (52), (58) and (59) we obtain the effective stiffness coefficients by the BDC-approach as:

$$
\begin{aligned}
& c_{11}^{\text {eff }}=\frac{\left\langle\sigma_{11}^{(I) e f f}\right\rangle_{B}}{\bar{\varepsilon}_{11}^{I}}, \quad c_{21}^{\text {eff }}=\frac{\left\langle\sigma_{22}^{(I) e f f}\right\rangle_{B}}{\bar{\varepsilon}_{11}^{I}}, \quad c_{61}^{\text {eff }}=\frac{\left\langle\sigma_{12}^{(I) e f f}\right\rangle_{B}}{\bar{\varepsilon}_{11}^{I}} \\
& c_{12}^{\text {eff }}=\frac{\left\langle\sigma_{11}^{(I I) e f f}\right\rangle_{B}}{\bar{\varepsilon}_{22}^{I I}}, \quad c_{22}^{\text {eff }}=\frac{\left\langle\sigma_{22}^{(I I) e f f}\right\rangle_{B}}{\bar{\varepsilon}_{22}^{I I}}, \quad c_{62}^{\text {eff }}=\frac{\left\langle\sigma_{12}^{(I I) e f f}\right\rangle_{B}}{\bar{\varepsilon}_{22}^{I I}} \\
& c_{16}^{\text {eff }}=\frac{\left\langle\sigma_{11}^{(I I I) e f f}\right\rangle_{B}}{2 \bar{\varepsilon}_{12}^{I I I}}, \quad c_{26}^{\text {eff }}=\frac{\left\langle\sigma_{22}^{(I I I) e f f}\right\rangle_{B}}{2 \bar{\varepsilon}_{12}^{I I I}}, \quad c_{66}^{\text {eff }}=\frac{\left\langle\sigma_{12}^{(I I I) e f f}\right\rangle_{B}}{2 \bar{\varepsilon}_{12}^{I I I}} .
\end{aligned}
$$

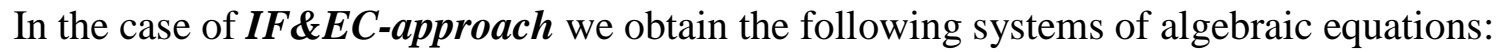

b.v.p. (I):

$$
\begin{aligned}
& {\left[(1-2 p) \bar{\varepsilon}_{11}-p \Delta \varepsilon_{11}^{m s}\right] c_{11}^{e f f}-p \Delta \varepsilon_{22}^{(I) m s} c_{12}^{\text {eff }}-2 p \Delta \varepsilon_{12}^{(I) m s} c_{16}^{\text {eff }}=(1-p)^{3}\left\langle\sigma_{11}^{(I) e f f}\right\rangle_{B}} \\
& {\left[(1-2 p) \bar{\varepsilon}_{11}-p \Delta \varepsilon_{11}^{m s}\right] c_{21}^{e f f}-p \Delta \varepsilon_{22}^{(I) m s} c_{22}^{e f f}-2 p \Delta \varepsilon_{12}^{(I) m s} c_{26}^{\text {eff }}=(1-p)^{3}\left\langle\sigma_{22}^{(I) e f f}\right\rangle_{B}} \\
& {\left[(1-2 p) \bar{\varepsilon}_{11}-p \Delta \varepsilon_{11}^{m s}\right] c_{61}^{e f f}-p \Delta \varepsilon_{22}^{(I) m s} c_{62}^{\text {eff }}-2 p \Delta \varepsilon_{12}^{(I) m s} c_{66}^{\text {eff }}=(1-p)^{3}\left\langle\sigma_{12}^{(I) e f f}\right\rangle_{B}}
\end{aligned}
$$

b.v.p. (II):

$$
\begin{aligned}
& -p \Delta \varepsilon_{11}^{(I I) m s} c_{11}^{e f f}+\left[(1-2 p) \bar{\varepsilon}_{22}-p \Delta \varepsilon_{22}^{(I I) m s}\right] c_{12}^{e f f}-2 p \Delta \varepsilon_{12}^{(I I) m s} c_{16}^{e f f}=(1-p)^{3}\left\langle\sigma_{11}^{(I I) e f f}\right\rangle_{B} \\
& -p \Delta \varepsilon_{11}^{(I I) m s} c_{21}^{e f f}+\left[(1-2 p) \bar{\varepsilon}_{22}-p \Delta \varepsilon_{22}^{(I I) m s}\right] c_{22}^{e f f}-2 p \Delta \varepsilon_{12}^{(I I) m s} c_{26}^{e f f}=(1-p)^{3}\left\langle\sigma_{22}^{(I I) e f f}\right\rangle_{B}
\end{aligned}
$$




$$
-p \Delta \varepsilon_{11}^{(I I) m s} c_{61}^{e f f}+\left[(1-2 p) \bar{\varepsilon}_{22}-p \Delta \varepsilon_{22}^{(I I) m s}\right] c_{62}^{e f f}-2 p \Delta \varepsilon_{12}^{(I I) m s} c_{66}^{e f f}=(1-p)^{3}\left\langle\sigma_{12}^{(I I) e f f}\right\rangle_{B}
$$

\section{b.v.p. (III):}

$$
\begin{aligned}
& -p \Delta \varepsilon_{11}^{(I I I) m s} c_{11}^{\text {eff }}-p \Delta \varepsilon_{22}^{(I I I) m s} c_{12}^{\text {eff }}+2\left[(1-2 p) \bar{\varepsilon}_{12}-p \Delta \varepsilon_{12}^{(I I I) m s}\right] c_{16}^{\text {eff }}=(1-p)^{3}\left\langle\sigma_{11}^{(I I I) e f f}\right\rangle_{B} \\
& -p \Delta \varepsilon_{11}^{(I I I) m s} c_{21}^{\text {eff }}-p \Delta \varepsilon_{22}^{(I I I) m s} c_{22}^{\text {eff }}+2\left[(1-2 p) \bar{\varepsilon}_{12}-p \Delta \varepsilon_{12}^{(I I I) m s}\right] c_{26}^{\text {eff }}=(1-p)^{3}\left\langle\sigma_{22}^{(I I I) e f f}\right\rangle_{B} \\
& -p \Delta \varepsilon_{11}^{(I I I) m s} c_{61}^{\text {eff }}-p \Delta \varepsilon_{22}^{(I I I) m s} c_{62}^{\text {eff }}+2\left[(1-2 p) \bar{\varepsilon}_{12}-p \Delta \varepsilon_{12}^{(I I I) m s}\right] c_{66}^{\text {eff }}=(1-p)^{3}\left\langle\sigma_{12}^{(I I I) e f f}\right\rangle_{B}
\end{aligned}
$$

Gathering Eqs. (71a), (72a) and (73a), we obtain the following system of equations for unknowns $\left(\begin{array}{lll}c_{11}^{\text {eff }} & c_{12}^{\text {eff }} & c_{16}^{\text {eff }}\end{array}\right)^{T}$ :

$[A]\left(\begin{array}{l}c_{11}^{\text {eff }} \\ c_{12}^{\text {eff }} \\ c_{16}^{\text {eff }}\end{array}\right)=(1-p)^{3}\left(\begin{array}{l}\left\langle\sigma_{11}^{(I) e f f}\right\rangle_{B} \\ \left\langle\sigma_{11}^{(I I) e f f}\right\rangle_{B} \\ \left\langle\sigma_{11}^{(I I I) \text { eff }}\right\rangle_{B}\end{array}\right)$

with $[A]=\left(\begin{array}{ccc}{\left[(1-2 p) \bar{\varepsilon}_{11}-p \Delta \varepsilon_{11}^{m s}\right]} & -p \Delta \varepsilon_{22}^{(I) m s} & -2 p \Delta \varepsilon_{12}^{(I) m s} \\ -p \Delta \varepsilon_{11}^{(I I) m s} & {\left[(1-2 p) \bar{\varepsilon}_{22}-p \Delta \varepsilon_{22}^{(I I) m s}\right]} & -2 p \Delta \varepsilon_{12}^{(I I) m s} \\ -p \Delta \varepsilon_{11}^{(I I I) m s} & -p \Delta \varepsilon_{22}^{(I I I) m s} & 2\left[(1-2 p) \bar{\varepsilon}_{12}-p \Delta \varepsilon_{12}^{(I I I) m s}\right]\end{array}\right)$

Similarly, from Eqs. (71b), (72b) and (73b), we obtain the system of algebraic equations for unknowns $\left(\begin{array}{ccc}c_{21}^{\text {eff }} & c_{22}^{\text {eff }} & c_{26}^{\text {eff }}\end{array}\right)^{T}$ :

$$
[A]\left(\begin{array}{c}
c_{21}^{\text {eff }} \\
c_{22}^{\text {eff }} \\
c_{26}^{\text {eff }}
\end{array}\right)=(1-p)^{3}\left(\begin{array}{l}
\left\langle\sigma_{22}^{(I) e f f}\right\rangle_{B} \\
\left\langle\sigma_{22}^{(I I) e f f}\right\rangle_{B} \\
\left\langle\sigma_{22}^{(I I I) e f f}\right\rangle_{B}
\end{array}\right)
$$

Finally, from Eqs. (71c), (72c) and (73c), we obtain the system of algebraic equations for unknowns $\left(\begin{array}{ccc}c_{61}^{e f f} & c_{62}^{e f f} & c_{66}^{e f f}\end{array}\right)^{T}$ :

$$
[A]\left(\begin{array}{c}
c_{61}^{e f f} \\
c_{62}^{e f f} \\
c_{66}^{e f f}
\end{array}\right)=(1-p)^{3}\left(\begin{array}{l}
\left\langle\sigma_{12}^{(I) e f f}\right\rangle_{B} \\
\left\langle\sigma_{12}^{(I I) e f f}\right\rangle_{B} \\
\left\langle\sigma_{12}^{(I I I) e f f}\right\rangle_{B}
\end{array}\right) .
$$




\section{NUMERICAL EXPERIMENTS}

\subsection{Circular pores}

In the case of circular pore we have results by 4 analytical and 2 numerical approaches. Comparison of such results for $c_{11}^{e f f}$ is shown if Fig.6. One pore is considered in the RVE and porosity is increased by increasing the radius of the pore.

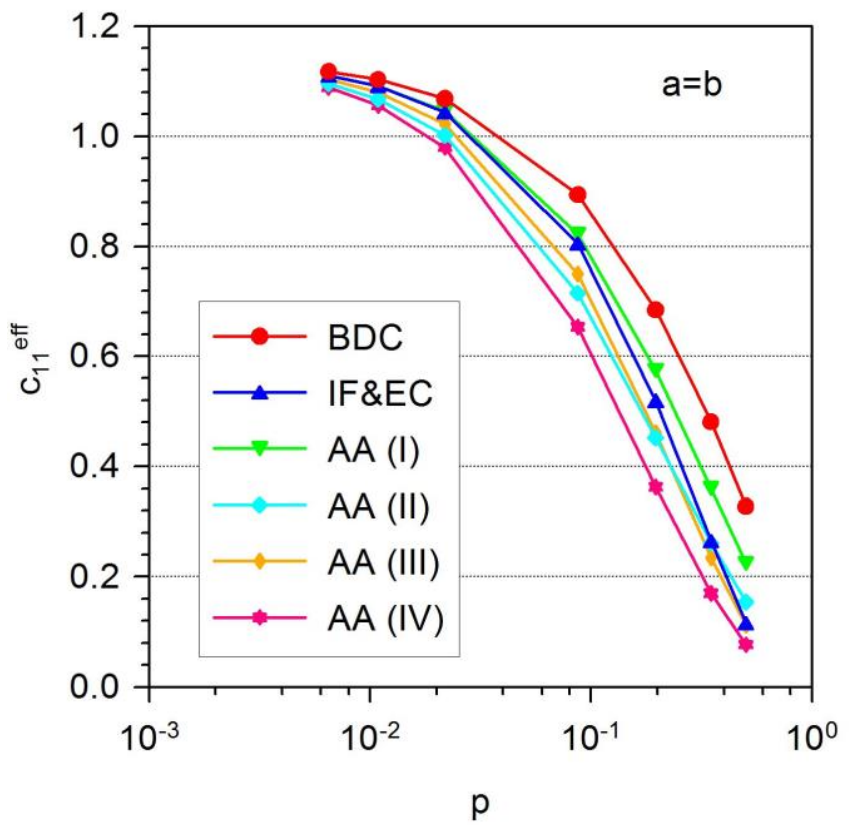

Fig. $6 c_{11}^{e f f}(p)$ by 6 various approaches

The effective material should be isotropic in the case of circular pores. The measure of anisotropy is shown in Fig.7
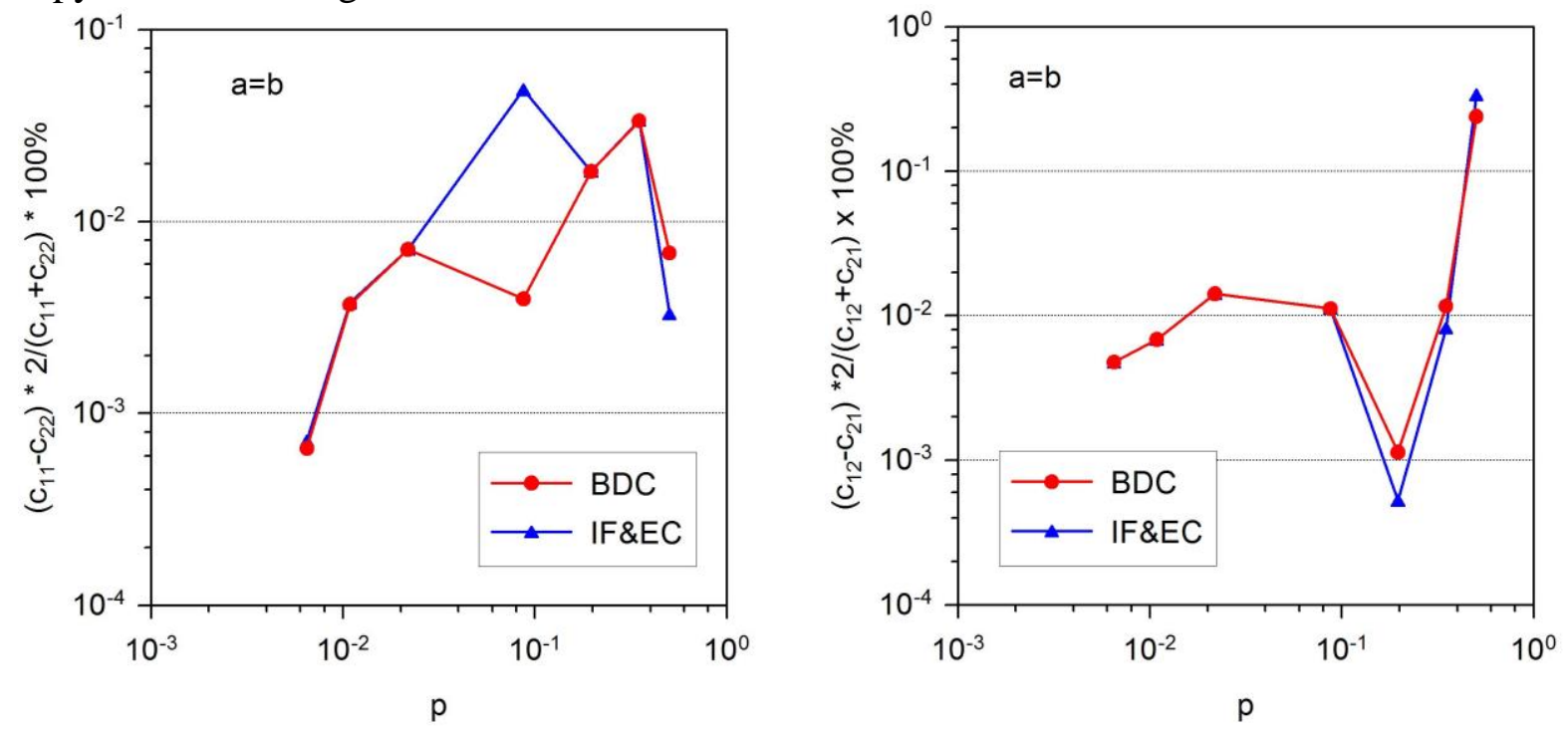


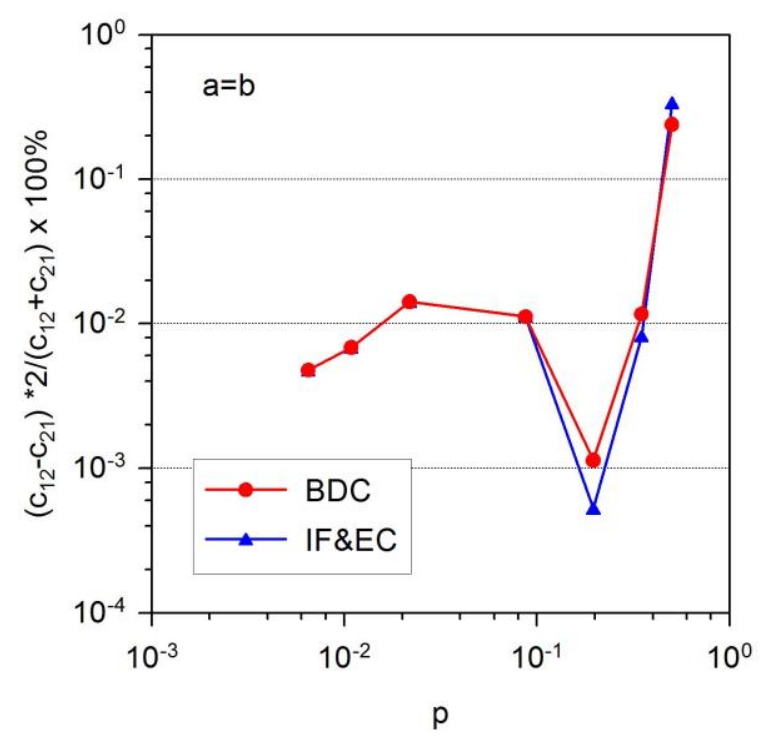

Fig.7 Percentage deviations from isotropy for stiffness coefficients of homogenized continuum with circular pores

Only numerical results are compared in this isotropy study because the analytical approaches exhibit perfect isotropy.

In numerical approaches, we have increased the porosity also by consideration of more pores in the RVE. Comparisons of numerical results obtained by modelling one and more pores are presented in Fig.8.
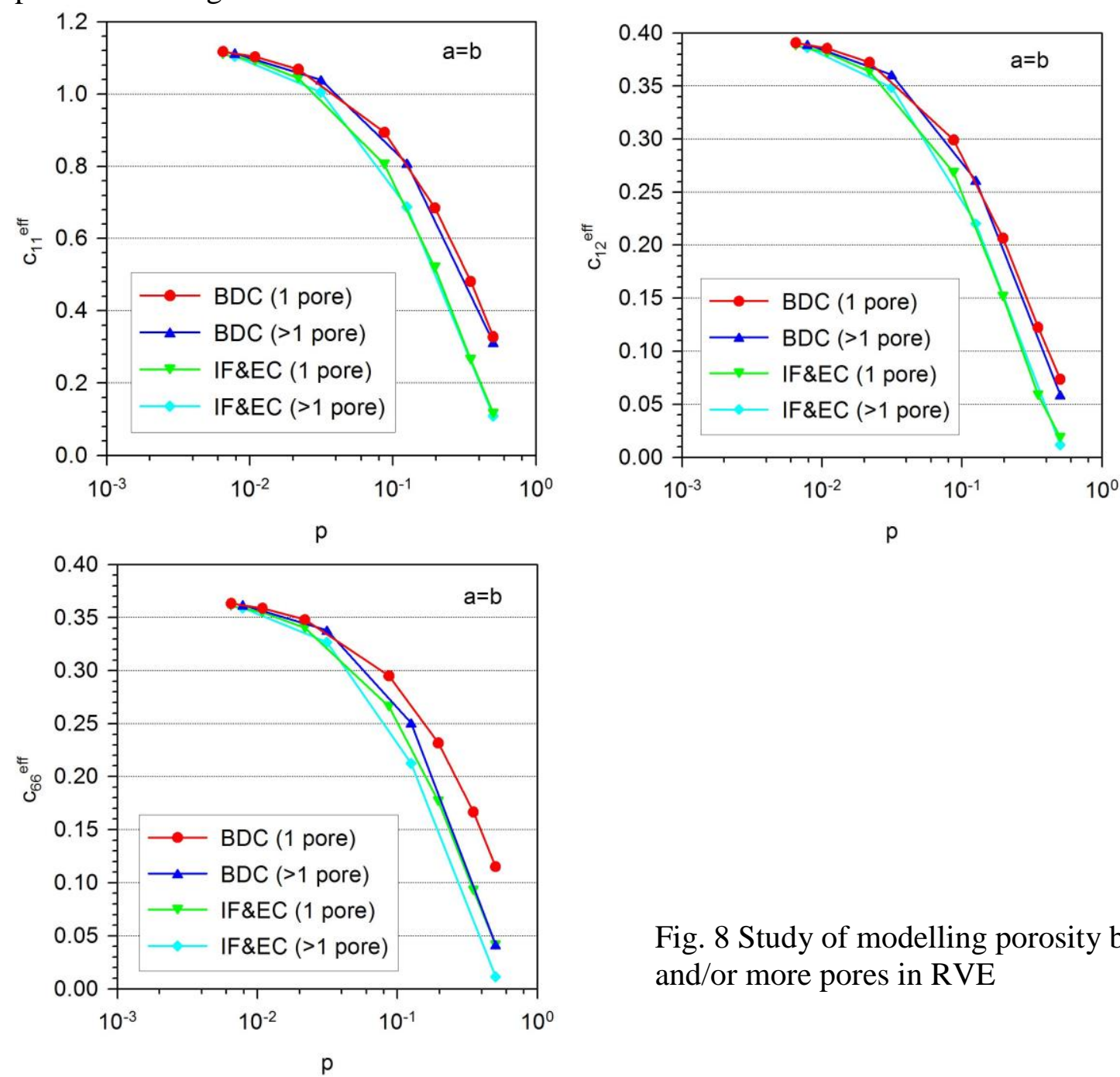

Fig. 8 Study of modelling porosity by one and/or more pores in RVE 
The independence of $c_{11}^{e f f}$ and $c_{12}^{\text {eff }}$ on the kind of modelling the increase of porosity demonstrates the theoretical expectation in the case of uniform distribution of pores. The interaction among the pores is involved into the model via the periodic boundary conditions even if only one pore is considered in the RVE. The deviations for $c_{66}^{\text {eff }}$ can be explained by very short distance of pores from the boundary of the RVE for higher values of porosity and rather small value of $c_{66}^{e f f}$.

\subsection{Elliptical pores}

Now we have numerical results by two approaches and analytical results only by AA(I) approach. Fig.9 shows comparison of results for $c_{11}^{\text {eff }}$ coefficient in the case elliptical pores with $a=2 b$
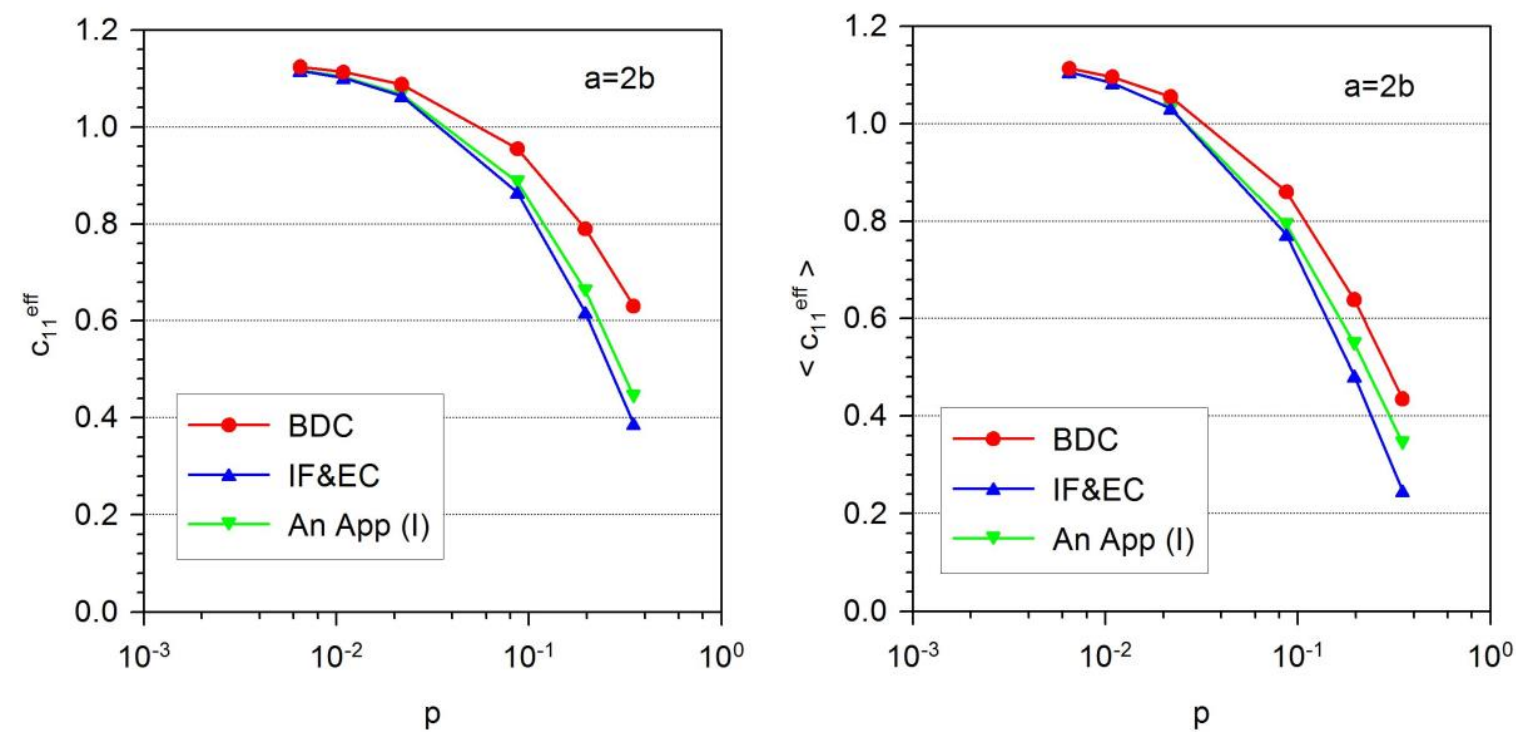

Fig. $9 c_{11}^{e f f}(p)$ for oriented and randomly oriented elliptical pores with $a=2 b$

The anisotropy in this case is verified remarkably for higher values of porosity as can be seen from Fig. 10
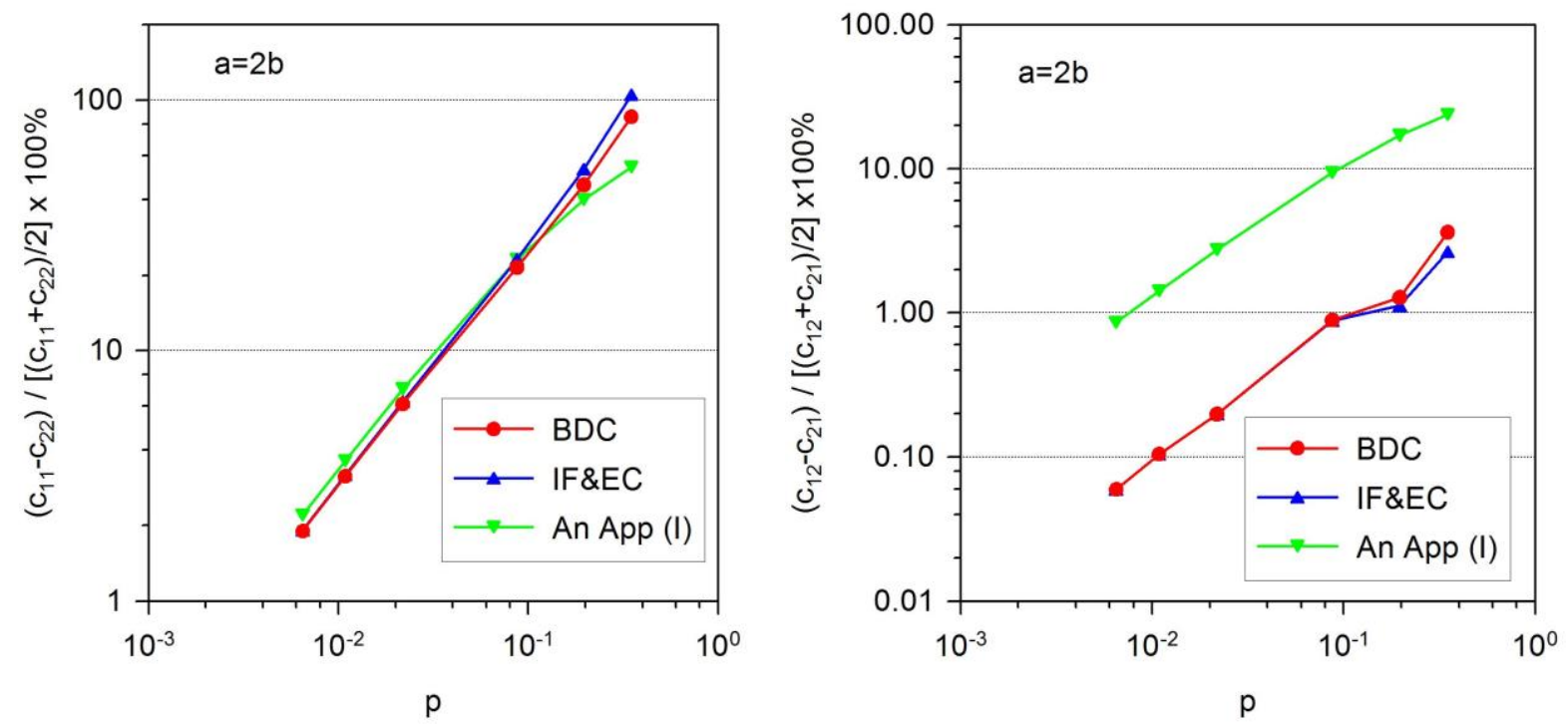


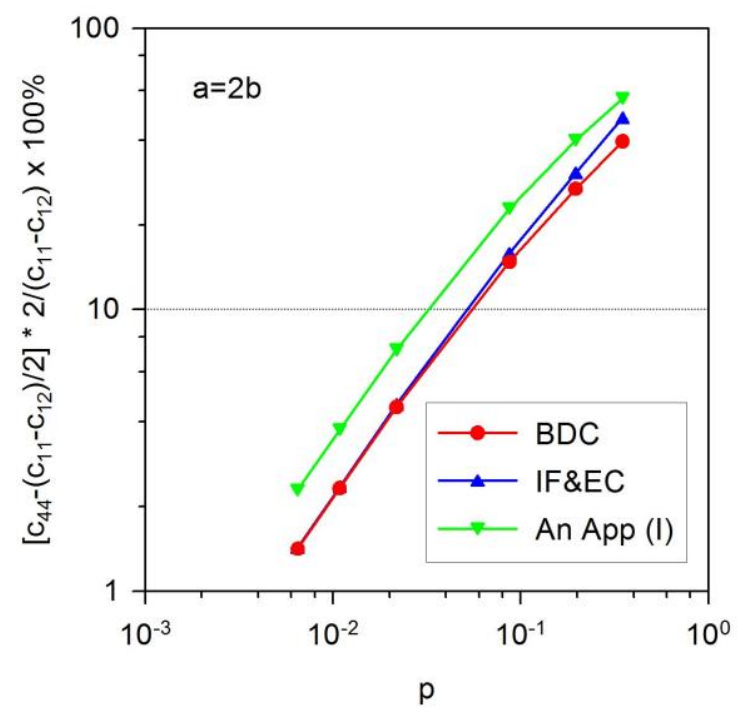

Fig. 10 Demostration of anisotropy in the case of oriented elliptic pores

The disturbance of orthotropic properties $\left(c_{12}^{\text {eff }} \neq c_{21}^{\text {eff }}\right)$ is rather weak even for high values of porosity.

In the case of IF\&EC approach the periodicity of boundary conditions is not guaranteed for homogenized bvp in contrast to the BDC approach. Therefore we can observe certain deviations between the results by IF\&EC approaches when the one and/or more pores are used for modelling porosity (see Fig. 11).
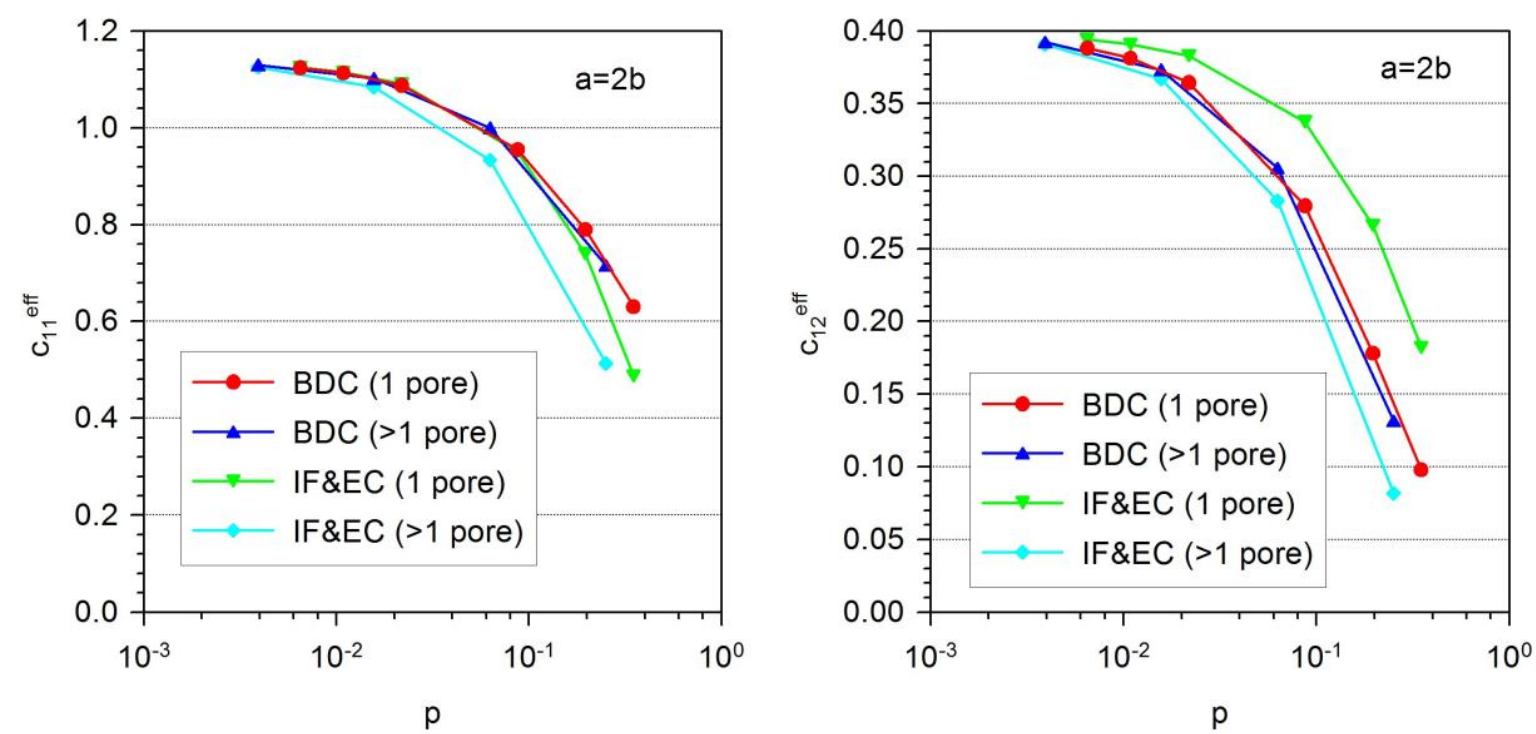

Fig. 11 Demonstration of dependence of results by IF\&EC approach on modelling of porosity

It can be seen from Fig. 12 that the solution of the micro-structural boundary value problem with 16 pores in the RVE exhibits the periodic symmetry. This symmetry is confirmed also by the BDC results, but it is not the case for homogenized problem of the IF\&EC approach 

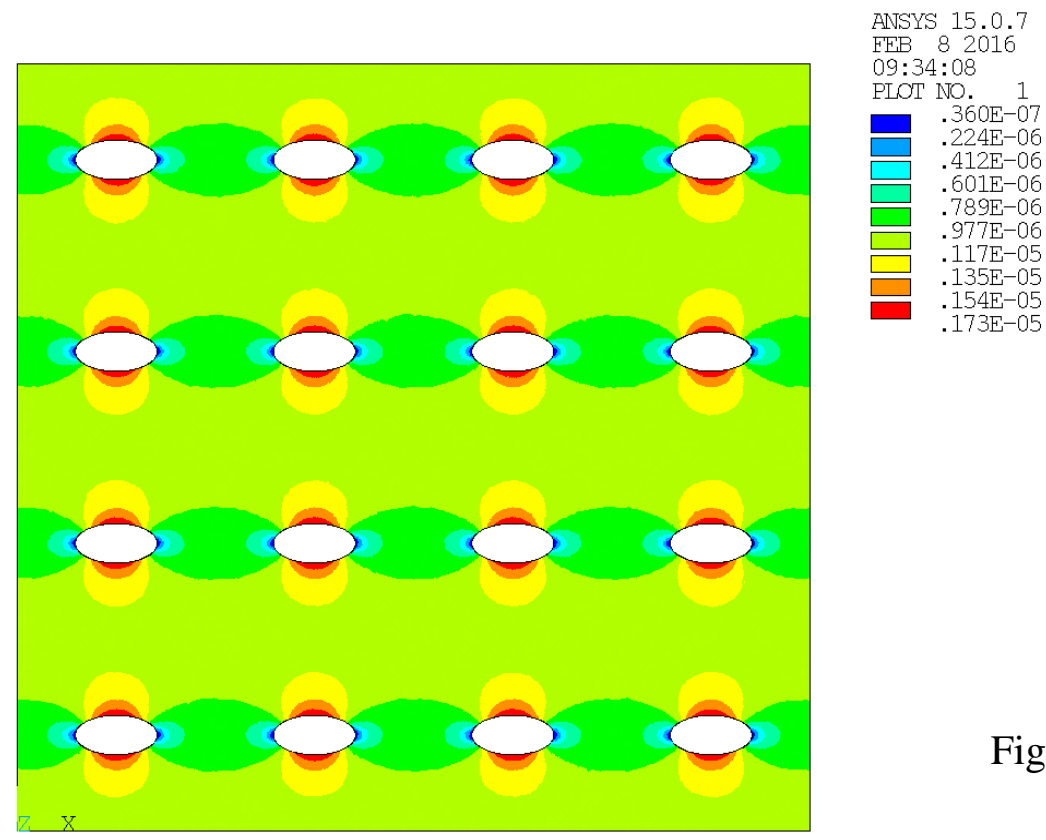

Fig. 12 Distribution of $\sigma_{11}^{(I) m s}$

\subsection{Application to plate bending problems}

For illustration, we present numerical results for bending of circular plate with a central circular hole subjected to uniform transversal loading, for which the exact solution is also available within the Kirchhoff-Love theory [11]. Fig. 13 shows the dependence of the effective Young modulus and Poisson ratio on the porosity with consideration of two shapes of pores.
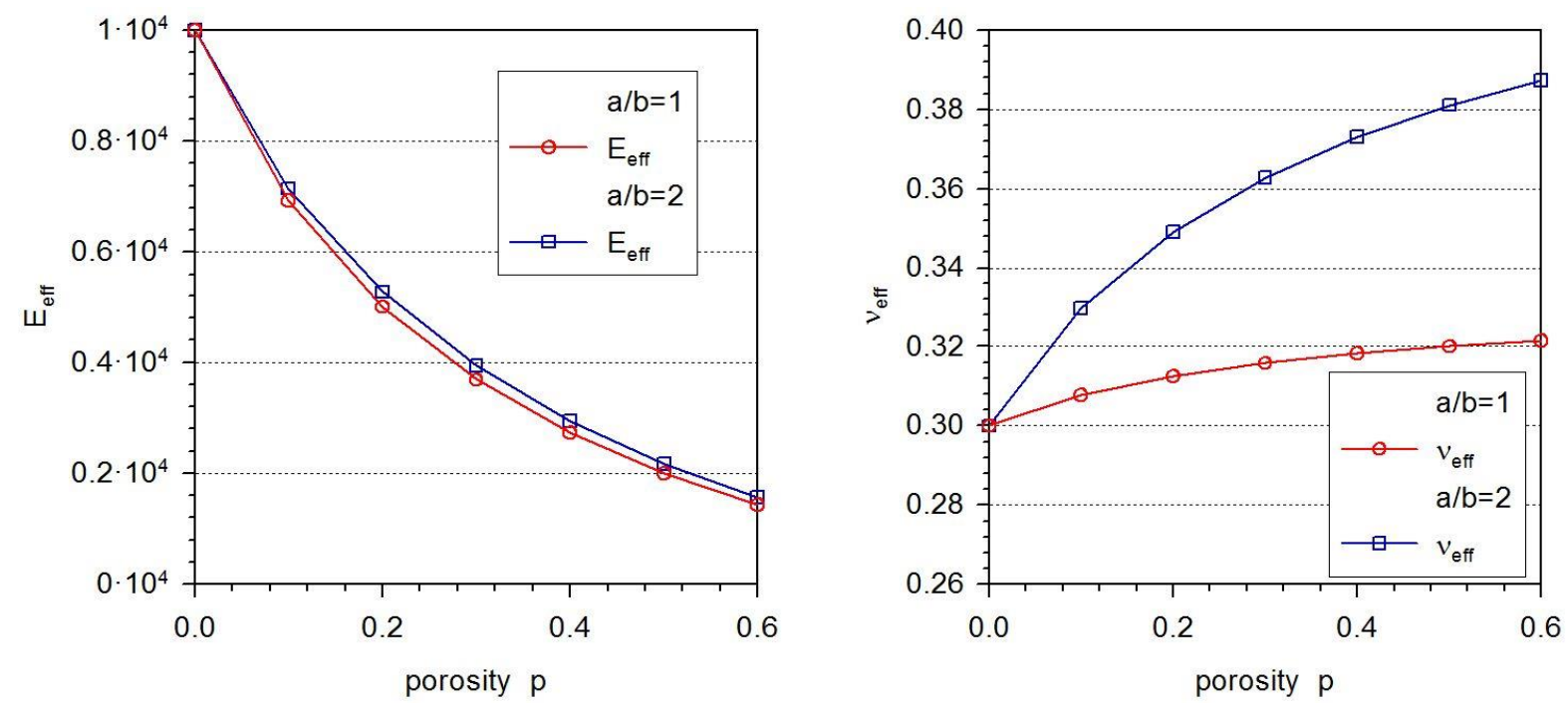

Fig. 13 The effective Young modulus and Poisson ratio vs porosity for circular pores $(a / b=1)$ and elliptic pores $(a / b=2)$

From Fig. 14, we can see a strong influence of the porosity on deflections of the plate.

The influence of the shape of pores on deflection can be seen from Fig. 15. 


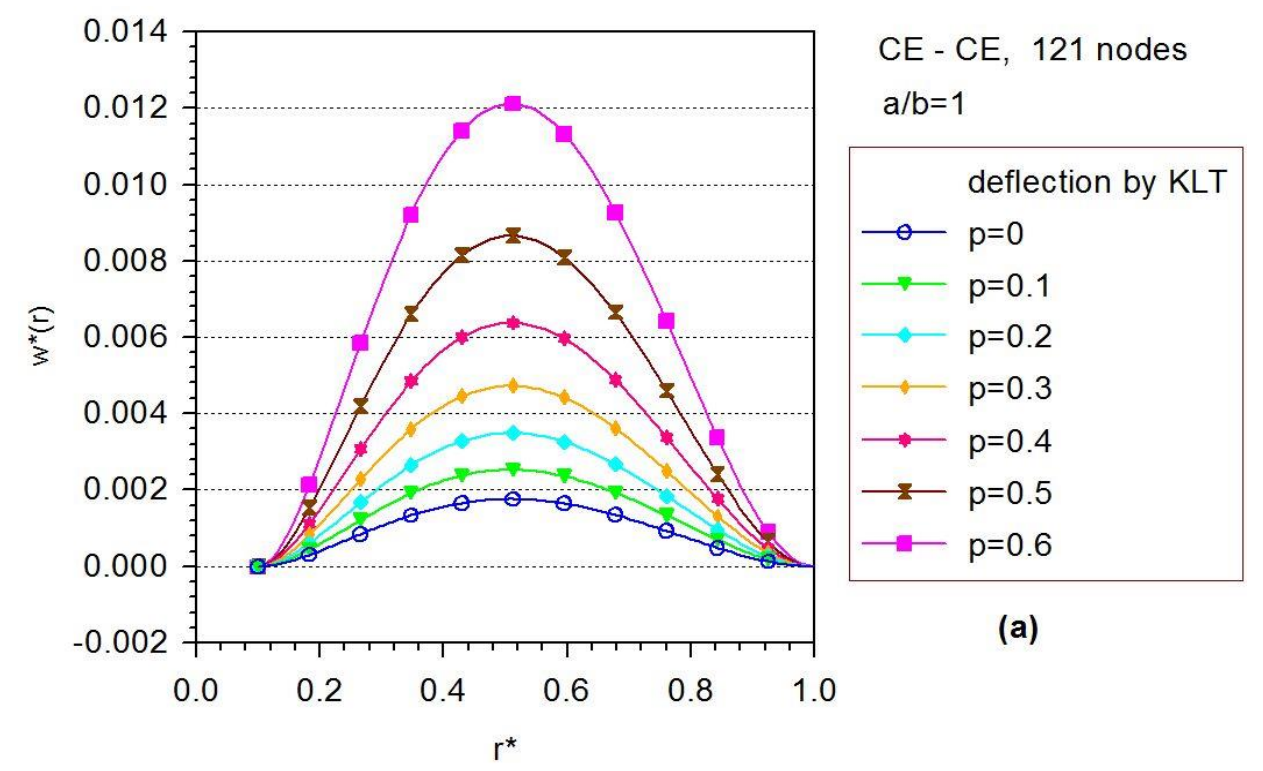

Fig. 14 The radial distribution of deflections in circular plates with various values of porosity

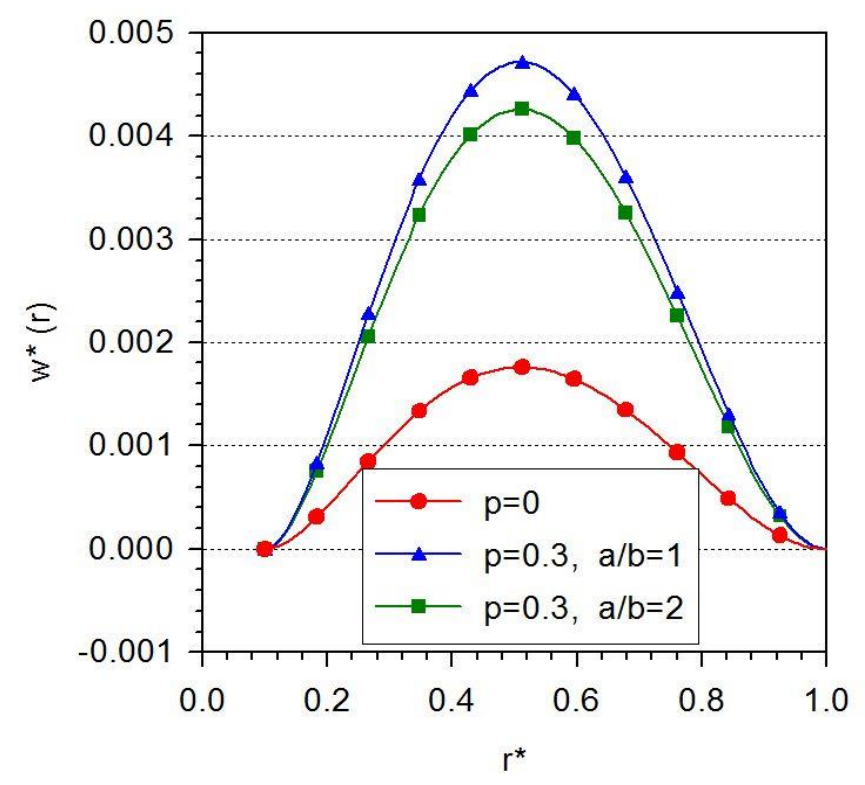

Fig. 15 Comparison of deflections in porous plates with two shapes of pores

It can be seen from Fig. 16 that the dependence of the maximal deflections on the porosity is nonlinear. It can be seen that the dependence is more expressive for thick plates. For thin plate $\left(r_{1}=50 h\right)$ all three theories give almost the same results, for the medium thickness $\left(r_{1}=10 h\right)$ there is coincidence only between the FSDPT $\left(1^{\text {st }}\right.$ order shear deformation plate theory) and the TSDPT ( $3{ }^{\text {rd }}$ order shear deformation plate theory), and finally for the thick plate $\left(r_{1}=5 h\right)$ a deviation can be seen even between the FSDPT and TSDPT results. The differences between the results by various theories are increasing with increasing the porosity. 


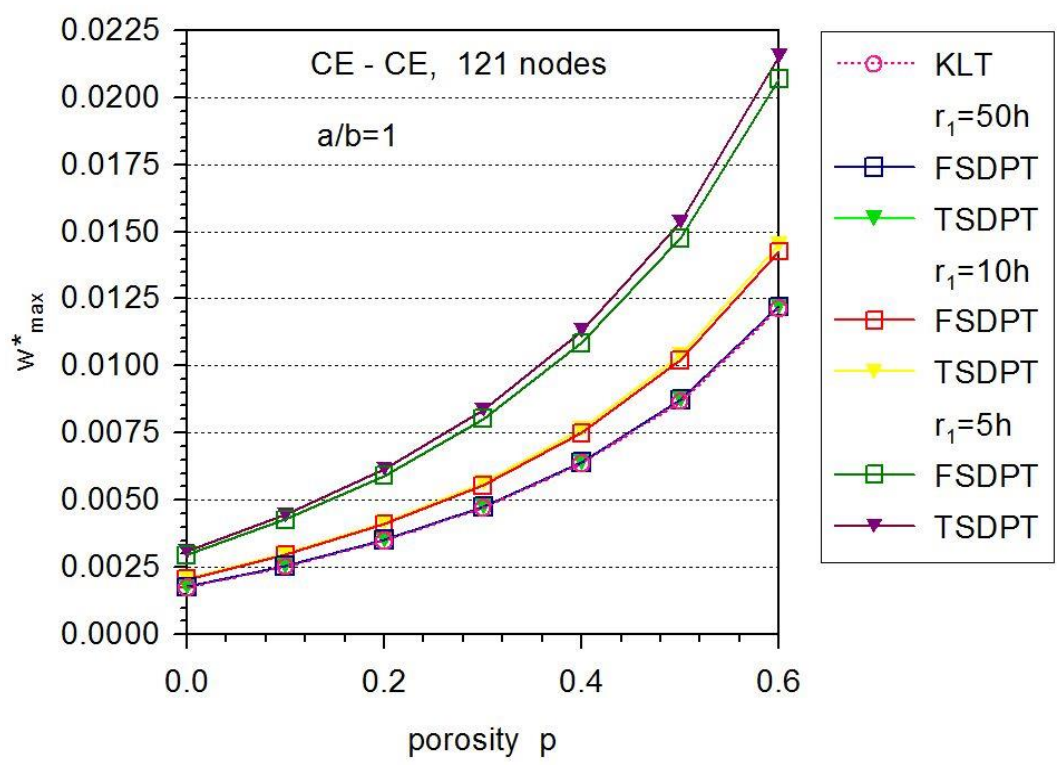

Fig. 16 Dependence of maximal deflections on the porosity

\section{CONCLUSIONS}

The homogenization of linear elastic media with empty pores is developed on the base of solution of micro-structural boundary value problems. In the case of uniformly distributed simple shape pores, such as circular and elliptic pores, we derived analytical formulae for compliances and/or stiffness coefficients of homogenized effective continuum. For the case of pores of arbitrary shape, we proposed two approaches for evaluation of material coefficients for effective homogenized continuum utilizing the solution of 3 appropriately selected boundary value problems. If the pores are uniformly distributed in the macrostructure, it is sufficient to select the RVE with one pore only. Various aspects of evaluation of effective coefficients are discussed in section devoted to numerical experiments. Finally, the obtained results for homogenization of porous media have been applied to study the influence of porosity on bending of elastic porous plates.

\section{REFERENCES}

[1] J.D. Eshelby, The determination of the elastic field of an ellipsoidal inclusion and related problems. Proc. Roy. Soc. London, A241, 376-396, 1957.

[2] Z. Hashin, S. Shtrikman, A variational approach to the theory of the elastic behavior of multiphase materials. J. Mech. Phys. Solids, 11, 127-140, 1963.

[3] R. Hill, A self-consistent mechanics of composite materials. J. Mech. Phys. Solids, 13, 213-222, 1965.

[4] T. Mori, K. Tanaka, Average stress in matrix and average elastic energy of materials with misfitting inclusions. Acta Metal., 21, 571, 1973.

[5] Y. Benveniste, A new approach to the application of Mori-Tanaka's theory in composite materials. Mechanics of Materials, 6, 147-157, 1987.

[6] T. Mura, Micromechanics of Defects in Solids. Martinus Nijhoff, 1982. 
[7] N.D. Cristescu, E.-M. Craciun, E.Soos, Mechanics of Elastic Composites. Chapman \& Hall/CRC, 2004.

[8] N.I. Muskhelishvili, Some Basic Problems of the Mathematical Theory of Elasticity. P. Noordhoff, 1953.

[9] L.I. Sedov, Mechanics of Continuous Media, Vol.2. World Scientific Publ. Co., 1997.

[10] A.I. Lurie, Theory of Elasticity. Springer, 2005.

[11] V. Sladek, J. Sladek, L. Sator, Physical decomposition of thin plate bending problems and their solution by mesh-free methods. Engineering Analysis with Boundary Elements, 37, 348-365, 2013. 